\title{
Tracing dynamics of laser-induced fields on ultrathin foils using complementary imaging with streak deflectometry
}

\author{
F. Abicht, ${ }^{1, \dagger}$ J. Braenzel, ${ }^{1}$ G. Priebe, ${ }^{2}$ Ch. Koschitzki, ${ }^{1}$ A. A. Andreev, ${ }^{1,3,4}$ \\ P. V. Nickles, ${ }^{1,5}$ W. Sander, ${ }^{1,6,7, *}$ and M. Schnürer ${ }^{1, \$}$ \\ ${ }^{1}$ Max-Born-Institut, Max-Born-Straße 2a, 12489 Berlin, Germany \\ ${ }^{2}$ XFEL GmbH, Notkestraße 85, 22607 Hamburg, Germany \\ ${ }^{3}$ Vavilov State Optical Institut, Birzhevaya line 12, 199064 St. Petersburg, Russia \\ ${ }^{4}$ St. Petersburg University, University emb.6, 199064 St. Petersburg, Russia \\ ${ }^{5}$ Center of Relativistic Laser Science, Institute for Basic Science, Gwangju 500-712, Republic of Korea \\ ${ }^{6}$ Technical University Berlin, Straße des 17. Juni 135, 10623 Berlin, Germany \\ ${ }^{7}$ ELI Delivery Consortium AISBL, Rue Montoyer 23, 1000 Brussels, Belgium
}

(Received 14 March 2016; published 8 September 2016)

\begin{abstract}
We present a detailed study of the electric and magnetic fields, which are created on plasma vacuum interfaces as a result of highly intense laser-matter interactions. For the field generation ultrathin polymer foils $(30-50 \mathrm{~nm})$ were irradiated with high intensity femtosecond $\left(10^{19}-10^{20} \mathrm{~W} / \mathrm{cm}^{2}\right)$ and picosecond $\left(\sim 10^{17} \mathrm{~W} / \mathrm{cm}^{2}\right)$ laser pulses with ultrahigh contrast $\left(10^{10}-10^{11}\right)$. To determine the temporal evolution and the spatial distribution of these fields the proton streak deflectometry method has been developed further and applied in two different imaging configurations. It enabled us to gather complementary information about the investigated field structure, in particular about the influence of different field components (parallel and normal to the target surface) and the impact of a moving ion front. The applied ultrahigh laser contrast significantly increased the reproducibility of the experiment and improved the accuracy of the imaging method. In order to explain the experimental observations, which were obtained by applying ultrashort laser pulses, two different analytical models have been studied in detail. Their ability to reproduce the streak deflectometry measurements was tested on the basis of three-dimensional particle simulations. A modification and combination of the two models allowed for an extensive and accurate reproduction of the experimental results in both imaging configurations. The controlled change of the laser pulse duration from 50 femtoseconds to 2.7 picoseconds led to a transition of the dominating force acting on the probing proton beam at the rear side of the polymer foil. In the picosecond case the $(\vec{v} x \vec{B})$-term of the Lorentz force dominated over the counteracting $\vec{E}$-field and was responsible for the direction of the net force. The applied proton deflectometry method allowed for an unambiguous determination of the magnetic field polarity at the rear side of the ultrathin foil.
\end{abstract}

DOI: 10.1103/PhysRevAccelBeams.19.091302

\section{INTRODUCTION}

Laser driven particle acceleration in plasmas is a wellestablished and still growing field of research. The possibility to accelerate particles over short distances allows for the construction of compact accelerators that have significant benefits for a variety of applications [1], as for example in basic sciences, material research and in the

\footnotetext{
*Deceased.

Corresponding author. abicht@mbi-berlin.de

Corresponding author. schnuerer@mbi-berlin.de

Published by the American Physical Society under the terms of the Creative Commons Attribution 3.0 License. Further distribution of this work must maintain attribution to the author $(s)$ and the published article's title, journal citation, and DOI.
}

biomedical sector. The lifetime and dynamics of the acceleration field depends on energy dissipation processes and thus is coupled to the duration of the laser pulse. Typically the time scale of these processes extends over several times the pulse duration, which results in highly transient acceleration field structures. Characterization of these fields is not only important to conclude about the plasma kinematics involved in the acceleration process but also to investigate the potential of cascaded acceleration schemes and the application of charged particle injection.

Here we study the strong fields on plasma vacuum interfaces generated on thin foils when irradiated with ultrahigh intensity femtosecond $\left(10^{19}-10^{20} \mathrm{~W} / \mathrm{cm}^{2}\right)$ and picosecond $\left(\sim 10^{17} \mathrm{~W} / \mathrm{cm}^{2}\right)$ laser pulses having the same energy content.

The focus of this investigation is not only on the target normal component of the electric field, which plays the key role in target normal sheath acceleration (TNSA) [2], but 
also on the radial electric field component (parallel to the foil surface) and the occurring toroidal magnetic field.

The present study develops new aspects to gain a comprehensive view on strong accelerating and deflecting fields in laser ion acceleration. A favorable combination of laser and target parameters was applied: for the generation of the investigated field structures ultrathin polymer foils $(30-50 \mathrm{~nm})[3,4]$ and ultrahigh contrast $\left(10^{10}-10^{11}\right)$ laser pulses were used. As previous experiments [5] have shown, these conditions allow for an optimization of the TNSA process [6,7]. In addition thin foils reduce the scattering of penetrating probe particles, which is a prerequisite for the applied proton imaging method [8].

To investigate the temporal evolution and the spatial distribution of the fields the proton streak deflectometry method [5] has been used and developed further. By applying the method in two imaging configurations we gained complementary information about the investigated field structure.

In this context the high laser contrast had a substantial influence: it increased the quality of the probing proton beam and led to a high reproducibility of the investigated field structure and hence of the whole experiment. In comparison with former experiments [5], consecutive proton imaging measurements now exhibited relatively low fluctuations when experimental conditions were kept constant. Therefore, the combination and comparison of images with different experimental parameters enables a qualitative and quantitative description of how the change of a specific parameter impacts on the laser induced field distribution.

In this paper several different experimental parameters were changed in consecutive measurements in order to monitor the associated induced change. The foil position with regard to the probing proton beam was varied systematically. This way the spatial extension of the fields could be investigated without decreasing the temporal and spatial resolution. A change of the laser pulse duration from the femtosecond to the picosecond range led to a transition of the dominating field action on the probing proton beam. In contrast to the femtosecond domain, in the picosecond domain the influence of the magnetic field was counteracting to the direction of the electric field and even more effective. Thus, the applied proton deflectometry method allowed for an unambiguous determination of the magnetic field polarity, which can provide insight to the field generating mechanisms.

In order to explain the experiments with femtosecond laser pulses, two different analytical models have been studied in detail. Their ability to reproduce the streak deflectometry measurements was tested on the basis of three-dimensional particle simulations by including the corresponding analytic field descriptions and accounting for the specific experimental conditions. As a result, specific but different characteristic features of the streak deflectometry measurements could be explained. Due to complementary streak deflectometry measurements with two different imaging configurations and using different experimental parameters a comprehensive investigation of the field structure becomes possible, such as the signature of a propagating proton front and the influence of the different magnetic and electric field components. This was not only useful to determine the application range and limitations of the single models, but also for the further development of the model descriptions itself. A modification and combination of the two models allowed for an extensive and accurate reproduction of the experimental results. This way, not only single and selected measurements could be reproduced by the particle simulation, but also (and at the same time) measurements with varied experimental parameters and measurements that were obtained using different imaging configurations. In this regard, the described methodological approach offers a new path for a comprehensive reconstruction of the spatial and temporal field distribution of laser-plasma interactions.

\section{EXPERIMENTAL SETUP}

The experiments have been conducted with the "High Field Laser" system at Max-Born-Institute, Berlin. It consists of two separate but optically synchronized Ti: sapphire amplifier chains, which are seeded with a shared Cross Polarized Wave (XPW)-frontend [9]. Due to the different architecture of these amplifier chains (arm A and B) laser pulses with different pulse duration and temporal contrast are generated. Laser arm A (100 TW) is based on a regenerative amplifier (inclusive spectral steering) and delivers pulses with 25 fs (FWHM of intensity) duration at an ASE background level of $10^{-10}-10^{-10}$. Laser arm B (70 TW) consists only of multipass amplifier sections and delivers pulses with 35 fs duration at an amplified spontaneous emission (ASE) background level of $10^{-11}-10^{-10}$. Respective third-order correlation measurements provide these values [9]. Applying pulses with this temporal contrast no degradation of fast ion emission with target foils as thin as $30 \mathrm{~nm}$ was observed which allowed omitting a plasma mirror in the beam line to achieve a necessary contrast level. Concerning previous experiments [5] the contrast level was increased by about 3 orders.

In the experiments described here, the (pump) laser pulses of laser arm $\mathrm{B}$ are used to generate a laser-matter interaction on a $30 \mathrm{~nm}$ polymer $\left(\mathrm{C}_{5} \mathrm{H}_{7} \mathrm{O}_{2}\right)$ foil $[3,4]$ with a solid state electron density $n_{e 0}$ of $3 \times 10^{23} 1 / \mathrm{cm}^{3}$ $\left(n_{e} \approx 300 n_{c}, n_{c}\right.$ is critical plasma density for light at $\approx 800 \mathrm{~nm}$ wavelength). The associated and fast evolving field structure of this interaction is investigated by means of a (probing) proton beam that is created with the help of laser arm A. This "probing" proton beam stems from laser irradiated titanium foils ( $5 \mu \mathrm{m}$ thick) and its adherent $\mathrm{CH}$ contamination. The increased temporal contrast improved the pointing stability of the probing proton beam significantly. Cutoff energies in the experiments extended up to 
$5 \mathrm{MeV}$. The shape of the kinetic energy distribution is exponentially decreasing. Proton numbers in the probing beam are sufficient to obtain single shot images with high contrast at about $50 \%$ of the cutoff energy. Absolute numbers were not determined. Estimation is possible with assumptions of laser to fast proton energy conversion and beam emission angles (cf. e.g. [6,7]).

In the presented cases, the focal intensity of the probe laser pulse (arm A) amounts to $\sim(1-5) \times 10^{19} \mathrm{~W} / \mathrm{cm}^{2}$ $\left(a_{0} \approx(2.2-5) ; a_{0}\right.$ is the relativistically normalized vector potential, variation depending on available laser system power of arm A). The focal intensity of the pump laser pulses depended on the setting of the laser pulse duration, which was varied between consecutive measurements by changing the position of the grating compressor. Only positively chirped pulses were applied. In our experiments the spectral bandwidth of the applied pump pulse was about $5 \%$ of the central wavelength. We can assume that within this limited bandwidth the response of the plasma to the incident laser radiation is similar. Therefore a chirp of an applied laser pulse (in case of a temporally stretched femtosecond pulse to picosecond duration) should not influence the interaction and only the resulting temporal width of the pulse matters. Furthermore all applied laser pulses used are shorter as the temporal resolution of the measurement setup in the experiments. Synchronization of pump and probe pulses is adjusted with a delay stage in the vacuum chamber of the experiment. Here, two cases are discussed. In the "femtosecond case" a 50 fs laser pulse with an intensity of $\sim 2 \times 10^{19} \mathrm{~W} / \mathrm{cm}^{2}$ $\left(a_{0} \approx 3.2\right)$ was applied and in the "picosecond case" a pulse length of $2.7 \mathrm{ps}$ was used leading to an intensity level of $\sim 6 \times 10^{17} \mathrm{~W} / \mathrm{cm}^{2} \quad\left(a_{0} \approx 0.55\right)$. The intensity $\left(I_{L}\right)$ calculation is based on measurements of pulse energy $\left(E_{L}\right)$, pulse duration $\left(\tau_{P}\right)$ and focal spot size $A_{L}$ (encircled area) with encircled energy content CCD-counts $_{\text {encircled area }} / \mathrm{CCD}$-counts overall area $_{\text {(cf. Fig. 1, }}$ number of integrated CCD-counts is determined above background): $I_{L}=E_{L} \cdot(\mathrm{CCD}$-counts encircled area $/$ CCD-counts overall area $) /\left(\tau_{P} A_{L}\right)$.

All target irradiation was done with p-polarized laser pulses using $f / 1.5$ off-axis parabolic mirrors. Microscopic on-line imaging of the attenuated beam focus was done prior the experiment. No degradation is observed in the case of applied stretched ps pulses. Examples of intensity distribution and temporal pulse shape are shown in Fig. 1.

The area used to determine the enclosed energy content is marked. Observation of slightly fluctuating pulse to pulse intensity distribution is estimated at a $20 \%$ level. Verification of the pulse duration as a function of compressor grating distance had been realized in former work. Second order correlation measurements showed temporal pulse envelopes without modulation (not published). The pulse energies are measured in front of the grating compressors. On target values are calculated with regard

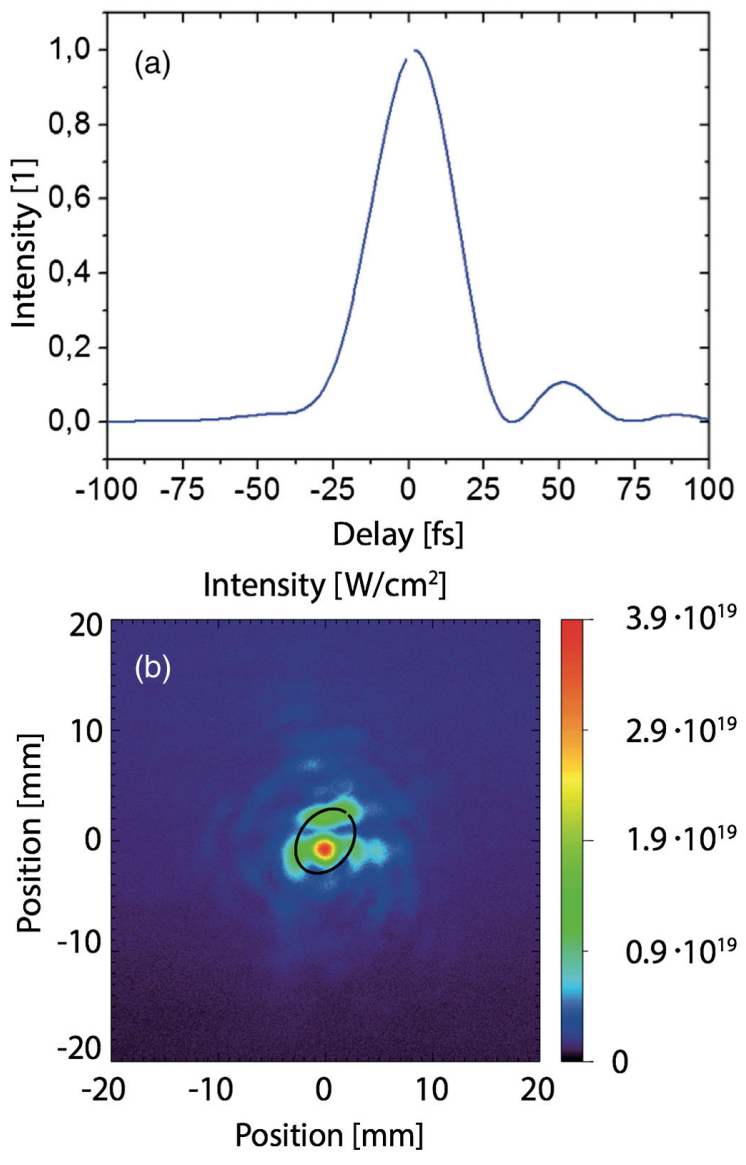

FIG. 1. Temporal pulse (a) shape and intensity distribution (b) (color coded, and marked area for determination of encircled energy content) used in experiments (example of laser probe beam).

to the measured relative transmissions of the grating compressors and beam lines inclusive focusing optics. Pulse durations of femtosecond and picosecond pulses are determined with a Spider and an Autocorrelator, respectively. The temporal profile with $50 \mathrm{fs}$ resolution and within a 200 ps temporal window was determined with a high dynamic range third order correlator measurement [9]. Beside apparent short prepulses we attribute the influence of the rising pulse front to a weak preplasma formation before the peak of the pulse arrives. This is indirectly implemented but not explicitly calculated in assumed conversion values between laser and target electron energy in all following discussions.

The experimental setups in longitudinal and transversal imaging configuration are illustrated in Figs. 2(a) and 2(b), respectively. The only difference between both setups consists in a rotation of the interaction (polymer) foil by $90^{\circ}$ while the incident angle of the pump laser pulse remains constant. Thus the angle of incidence at the target to probe is changed from $45^{\circ}$ to $-45^{\circ}$ in reference to the target normal. Therefore the orientation of the laser induced field distribution in space is both rotated (by $90^{\circ}$ around the 


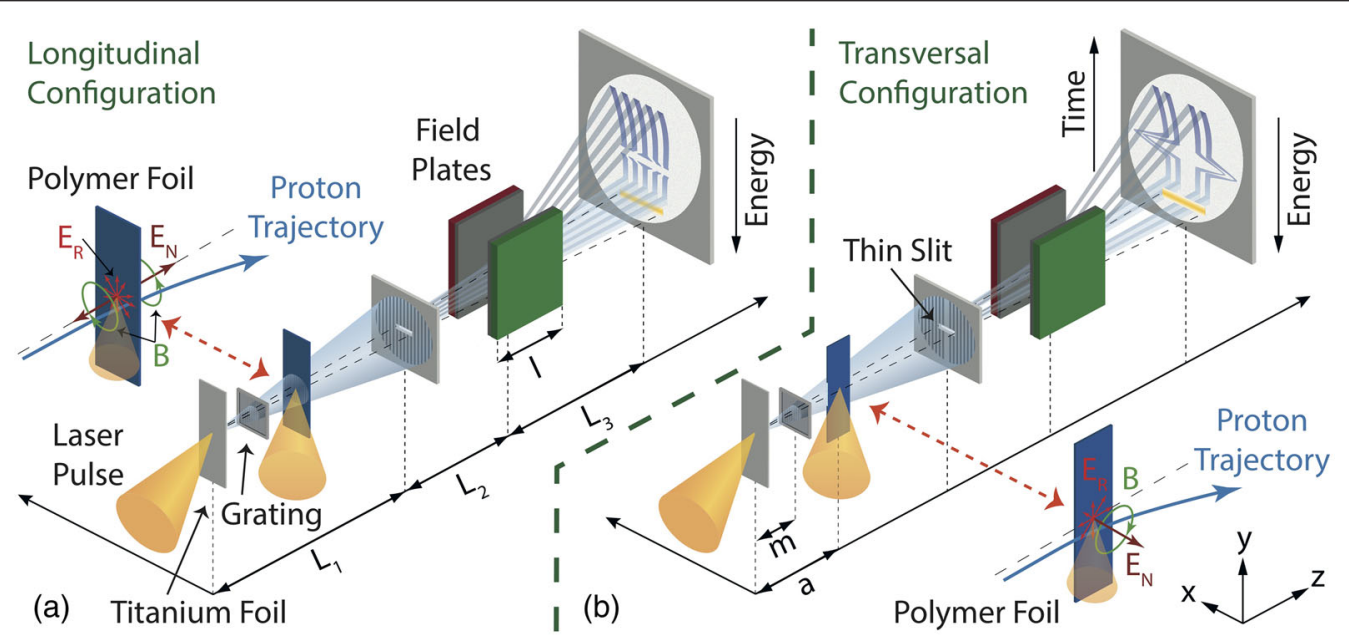

FIG. 2. Setup and imaging configurations: (a) longitudinal configuration, (b) transversal configuration.

$\mathrm{y}$-axis) and mirrored (along the x-axis). However, if the laser parameters are identical in both cases (and spot symmetry is assumed), the induced field structure itself is not changed, only its absolute orientation in space. On their way to the detector the probing protons propagate either parallel (longitudinal configuration) or perpendicular (transversal configuration) to the surface normal of the polymer foil (interaction target) and are deflected by the induced fields due to the acting Lorentz force. By inserting a beam mask (grating) the deflection along the x-direction becomes detectable. However, depending on the imaging configuration the induced proton deflection is dominated by different components, as illustrated in Figs. 2(a) and 2(b). Here $E_{\mathrm{N}}$ indicates the main electric field component normal to the target surface, $E_{\mathrm{R}}$ the radial electric field component and $B$ the toroidal magnetic field.

By measuring the energy dependent proton deflection in both imaging configurations complementary information on the fast evolving field structure is obtained. For this purpose a modified Thomson spectrometer [5] was applied. This method is called "proton streak deflectometry" [5] and is explained in detail in Refs. [5,10].

The entrance slit has a width of $288 \mu \mathrm{m}$ and a length of about $1 \mathrm{~cm}$. The used distances in Fig. 2 are $L_{1}=477 \mathrm{~mm}$, $L_{2}=228 \mathrm{~mm}, L_{3}=515 \mathrm{~mm}, a=35 \mathrm{~mm}$, and $m=20 \mathrm{~mm}$. The applied magnet has the length $l=50 \mathrm{~mm}$ and corresponding to this extension the effective field strength $B_{\mathrm{x}}=0.34 \mathrm{~T}$. The field of the spectrometer magnet was completely mapped in 3D. Concerning the obtained smooth field variation the deflectograms [Figs. 3(a)-3(d)] were processed using a graphic rectification algorithm (cf. details in [10]). As a detector a multichannel plate (MCP) from Hamamatsu (Type F1942-04) coupled with a phosphor screen was used in combination with an attached camera. The imaging quality of the MCP was verified with laser accelerated proton beams and projection imaging of test objects and the energy dependent ion sensitivity was calibrated [11]. The overall energy resolution $\Delta \epsilon$ of the
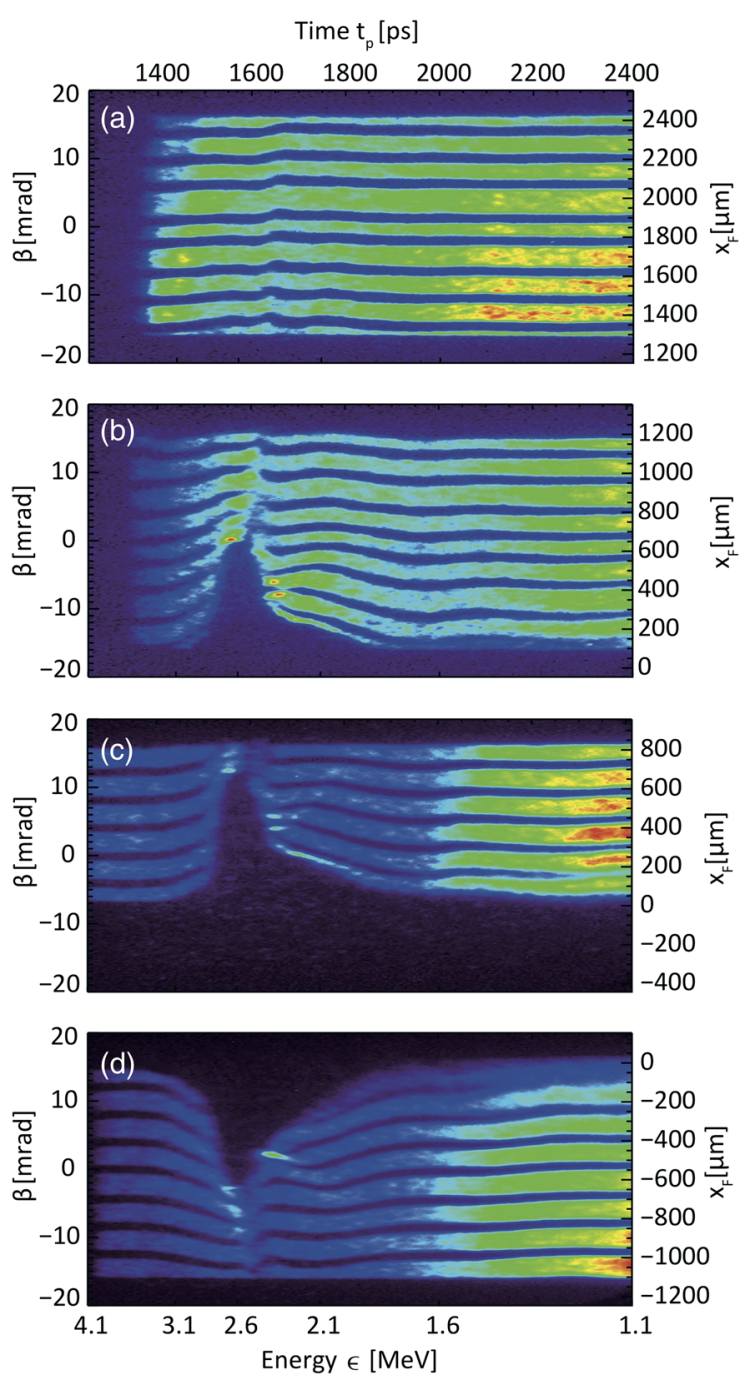

FIG. 3. Proton streak deflectometry measurements in transversal imaging configuration. The absolute x-position $\left(d_{x}\right)$ of the polymer foil was changed in consecutive shots: (a) $d_{x}=-1854 \mu \mathrm{m}$, (b) $d_{x}=-654 \mu \mathrm{m}$, (c) $d_{x}=-254 \mu \mathrm{m}$, (d) $d_{x}=545 \mu \mathrm{m}$. 
imaging system was approximately $45 \mathrm{keV}$ for proton energies around $2.5 \mathrm{MeV}$. In general the relative energy resolution is better than 3\% of the relevant kinetic energy values. In order to exclude contributions of other ion species besides protons the MCP was temporally gated. By switching off the voltage between the phosphor screen and the MCP, at a time $130 \mathrm{~ns}$ after the laser-plasma interaction, a pure proton signal with energies above $0.5 \mathrm{MeV}$ could be detected.

In transversal configuration, the temporal resolution $\Delta t$ is around $30 \mathrm{ps}$ if an interaction length of $l_{1}$ of $100 \mu \mathrm{m}$ is assumed [10]. In this configuration two additional Thomson spectrometers were installed opposite the front and rear surface of the polymer foil. These spectrometers were applied to record the energy distribution of the individual ion species that are accelerated in opposite directions normal to the surface of the interaction target.

\section{EXPERIMENTAL RESULTS}

Figures 3(a)-3(d) show a selection of processed streak deflectometry measurements, which were obtained in transversal imaging configuration. Different regions of the laser-induced fields were probed by changing the $\mathrm{x}$ position of the polymer foil with regard to the probing proton beam in consecutive shots. The measured proton density distributions $\rho(x, y)$ on the MCP have been processed by means of a numerical coordinate transformation method that is presented in detail in Ref. [10]. As a result the distributions $\tilde{\rho}\left(\beta, t_{p}\right)$ can be visualized as a function of the initial proton emission angle $\beta$ (in the $\mathrm{x}-\mathrm{z}$ plane) and the probing time $t_{p}$, which are represented by the upper abscissa and the left ordinate, respectively. The lower abscissa indicates the corresponding proton energy for $\beta=0$. The right ordinate shows the relative lateral distance $x_{f}$ (in the $\mathrm{x}$-direction) between a proton and the focus position at the moment in time, when the proton passes through the $\mathrm{x}-\mathrm{y}$ plane at $z=a$ [cf. Fig. 2(b)]. The lateral focus distance $x_{f}$ depends both on the initial emission angle of the proton and the x-position of the laser focus on the polymer foil. Proton traces in Fig. 3 are characterized with an $\mathrm{x}$-coordinate $\mathrm{x}_{F}$ which is the relative distance between the probing proton and the target surface along the $\mathrm{x}$-axis [cf. Fig. 2(b)]. The absolute and varied position of the target foil concerning the $\mathrm{x}$-coordinate is designated with $d_{x}$ (cf. Fig. 3). Changing $d_{x}$ results in different $x_{f}$ for a fixed detector and probe beam position. However, the definition of the lateral focus distance $x_{f}$ holds only for an idealized proton, which is not deflected by the laser-induced fields. Due to the action of the Lorentz force, the trajectory of a proton can be altered in the extended field region around the polymer foil. Thus the final propagation angle of a proton (in the $\mathrm{x}-\mathrm{z}$ plane) might differ from its initial ejection angle $\beta$. For this reason the lateral focus distance $x_{f}$ is only an approximation of the real distance, if a proton is deflected in the laser induced field region around $z=a$.

The arrival of the laser pulse at the titanium target initiates the creation of the probing proton beam and defines the absolute time zero. By means of an optical delay stage within the experimental chamber the arrival time $t_{\text {pump }}$ of the laser pump pulse at the polymer target was adjustable with respect to time zero. Thus, the time $t_{\text {pump }}$ defines the arrival time of the pump laser pulse at the polymer target with regard to the creation of the probing proton beam at the titanium target. In addition, the time $t_{\text {pump }}$ can be regarded as the arrival time of protons at the polymer target, which are accelerated at the titanium target at time zero and propagate with the specific energy $\epsilon=\epsilon_{\text {pump. }}$. In fact this relation is the definition of the socalled t-pump energy $\epsilon_{\text {pump. }}$. In other words a proton with the kinetic energy $\epsilon=\epsilon_{\text {pump }}$ needs the time $t_{p}=t_{\text {pump }}$ to propagate from the titanium to the polymer target.

In the presented experiments the time $t_{\text {pump }}$ is set to $1571 \mathrm{ps}$, which corresponds to the proton t-pump energy $\epsilon_{\text {pump }}=2.6 \mathrm{MeV}$. With the generation of the electric fields, the probing protons (with energies around $\epsilon \approx \epsilon_{\text {pump }}$ ) are deflected away from the surfaces of the interaction target depending on their relative position, propagation direction and energy. The temporal dependence of the deflection can be divided in several intervals.

Protons with energies $\epsilon>\epsilon_{\text {pump }}$ reach the polymer target $(z=a)$ even before the laser pump pulse impinges on its surface. The initiated fields only affect a proton if its relative distance to the interaction center (pump focus) is less than the radial extension of the field distribution in the Z-direction.

In order to visualize the influence of transversal force components (in the x-direction, i.e. perpendicular to the propagation direction of the probing proton beam) a grating with 300LPI (lines per inch) was used to intersect the proton beam (cf. Fig. 2) into single beamlets. Each of these beamlets consists of protons that are ordered in time and space (in propagation direction) according to their energy $\epsilon$. In addition, each beamlet can be attributed to protons having initial ejection angles of a certain interval. Depending on their energy, the final propagation angles of these protons can be changed during the interaction with the laser-induced fields. Correspondingly, the mean propagation angle of a whole beamlet can be altered for a certain energy interval and might differ from its initial ejection angle $\beta$. The thin slit confines the beamlets further, allowing only for protons propagating close to the $\mathrm{x}-\mathrm{Z}$ plane. Thus, only deflections in $\mathrm{x}$-direction can be measured. Within the permanent magnetic field of the Thomson spectrometer protons are dispersed in y-direction according to their energy. On the detector plane (x-y plane), the energy distribution of each beamlet is transformed into a spatial distribution along the y-direction and becomes visible in the form of proton traces (proton streaks). The 
time dependent action of transverse force components leads to deflections of these traces, i.e. streak deflections. Regarding the processed images of Figs. 3, 5, 6, 8, 9 and 11 the streak deflections are shown as deviations from the initial emission angle $\beta$. The value of the initial emission angle $\beta$ that corresponds to a certain proton trace is given by its relatively constant position for energies $\epsilon \gg \epsilon_{\text {pump. }}$. These energies correspond to probing times $\left(t_{p} \ll t_{\text {pump }}\right)$ well before the deflecting fields are initiated. Thus, the propagation angles of protons with such energies are not altered by these fields and are equal to their initial emission angles. The dependence of the initial emission angle on energy during the acceleration process at the titanium foil has been investigated in Ref. [5]. For the energy range considered here, this dependency in negligible with regard to the induced deflections around the polymer foil.

In view of all measurements, the deflected traces exhibit a pronounced deflection symmetry, indicating a highly symmetric field distribution. Protons passing the interaction target on different sides [cf. Figs. 3(c) and 3(d)], but with similar energies $\epsilon_{p}$ and similar initial absolute values of the lateral focus distance $\left|x_{F}\right|$, show similar deflection amplitudes. On both sides the deflection strongly depends on the initial distance to the target surface. Protons passing closer to the foil surface are deflected stronger than protons, which pass at a larger distance. The time $t_{\text {pump }}$ defines the arrival time of the pump laser pulse at the polymer foil with regard to the creation of the probing proton beam at the titanium target.

As shown in Figs. 3(c) and 3(d) the deflection becomes visible at the time $t_{f}=t_{p}=1410 \mathrm{ps}$ and can be characterized by a steep increase. Proton traces with lateral focus distances $\left|x_{F}\right| \lesssim 650 \mu \mathrm{m}$ (for $t_{p} \leq t_{\text {pump }}$ ) show a deflection maximum at the time moment, which corresponds exactly to the setting of the pump time $t_{\text {pump }}=1571 \mathrm{ps}$. For traces at higher distances $\left(\left|x_{F}\right| \gtrsim 650 \mu \mathrm{m}\right)$ the deflection maximum is shifted towards lower proton energies. After the maximum the deflection decays relatively quickly for traces with $\left|x_{F}\right| \lesssim 650 \mu \mathrm{m}$, on a time scale of approximately 80 ps. Then the decay flattens and within 250 ps the deflection declines to a quasiconstant value. At higher distances $\left(\left|x_{F}\right| \gtrsim 400 \mu \mathrm{m}\right)$ the deflected traces exhibit a second local maximum before dropping to constant values in time. At this point the proton deflection becomes relatively independent on the initial ejection angle $\beta$. The time $t_{f} \approx 1410 \mathrm{ps}$ when the deflection becomes visible is connected with the energy $\epsilon_{f} \approx 3.23 \mathrm{MeV}$ of the fastest influenced protons, as illustrated in Fig. 3(c).

The corresponding velocity value can be used to estimate the extension of the electric field in the z-direction under the assumption that the electric field component in the $\mathrm{z}$-direction (along the surface) is negligible. In the case of Fig. 3(c) an extension radius $r_{f}$ of $4.3 \mathrm{~mm}$ is obtained. Considering that the foil target has a total extension of
$5 \mathrm{~mm}$ in the z-direction and is irradiated in its center, the deduced extension radius is relatively high. This indicates that radial field components along the foil surface (in the z-direction) cannot be neglected in the presented case. Using the extension of the foil in the z-direction as an upper limit of the radial field extension, the time resolution of the method can be estimated. At the probing time $t_{p}=t_{\text {pump }}$ the time resolution $\Delta t$ is approximately $250 \mathrm{ps}$.

Simultaneously registered ion spectra from front and rear side emission of the polymer foil target are nearly identical (cf. Fig. 4) and confirm the symmetry of Figs. 3(c) and 3(d).

Figures 3(a) and 3(b) show the recorded streak deflections, which correspond to protons passing the foil surface at larger distances at the time $t_{\text {pump }}$, i.e. for higher values of $\left|x_{F}\right|$. Proton streak deflections are visible up to the maximum-recorded value $\left|x_{F}\right| \approx 2400 \mu \mathrm{m}$, which is a measure of the effective field extension normal to the foil surface. Thus, for the electric field component $E_{\mathrm{N}}$ normal to the target surface an effective extension length $l_{E \mathrm{n}} \gtrsim$ $2400 \mu \mathrm{m}$ is found. For distances $\left|x_{F}\right| \gtrsim 700 \mu \mathrm{m}$ the recorded proton streaks do not overlay and are clearly distinguishable, even at the deflection maximum. For these distances the deflection amplitude is smaller than the width of a proton streak $(\approx 50 \mu \mathrm{m})$ and decreases at higher values of $\left|x_{F}\right|$. This dependency could be explained by a field gradient $\partial E / \partial x$, which becomes relatively flat for larger distances $\left(\left|x_{F}\right| \gtrsim 700 \mu \mathrm{m}\right)$. If protons of adjacent streaks are affected by almost the same force, the amplitude of their deflection becomes similar and different streaks cannot overlay on the detector. A careful analysis of the presented
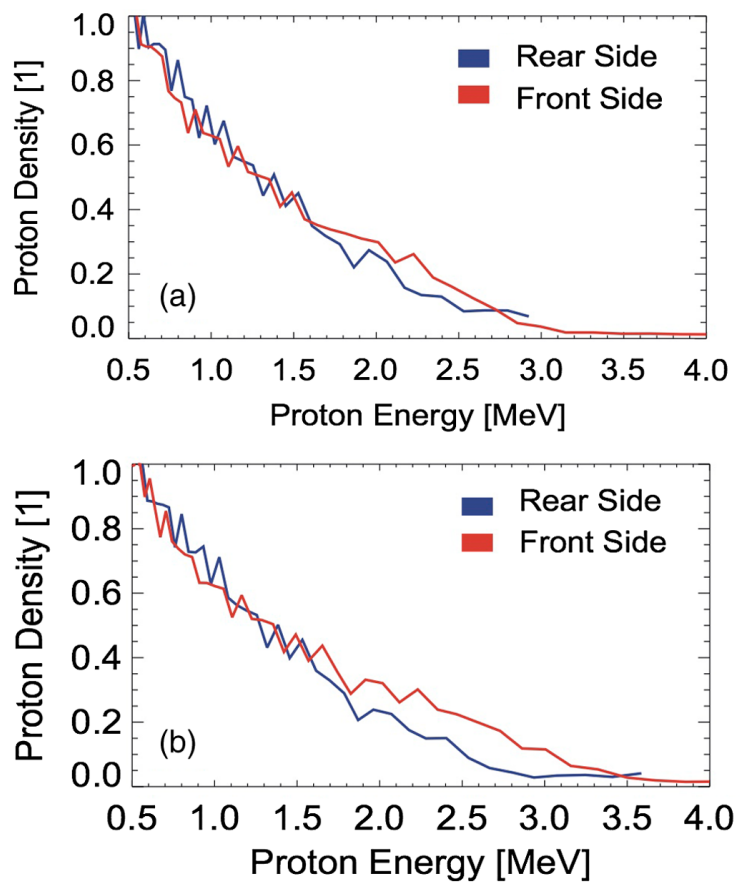

FIG. 4. Measured proton spectra corresponding to the deflectograms of Fig. 3(c) [spectrum (a)] and Fig. 3(a) [spectrum (b)]. 

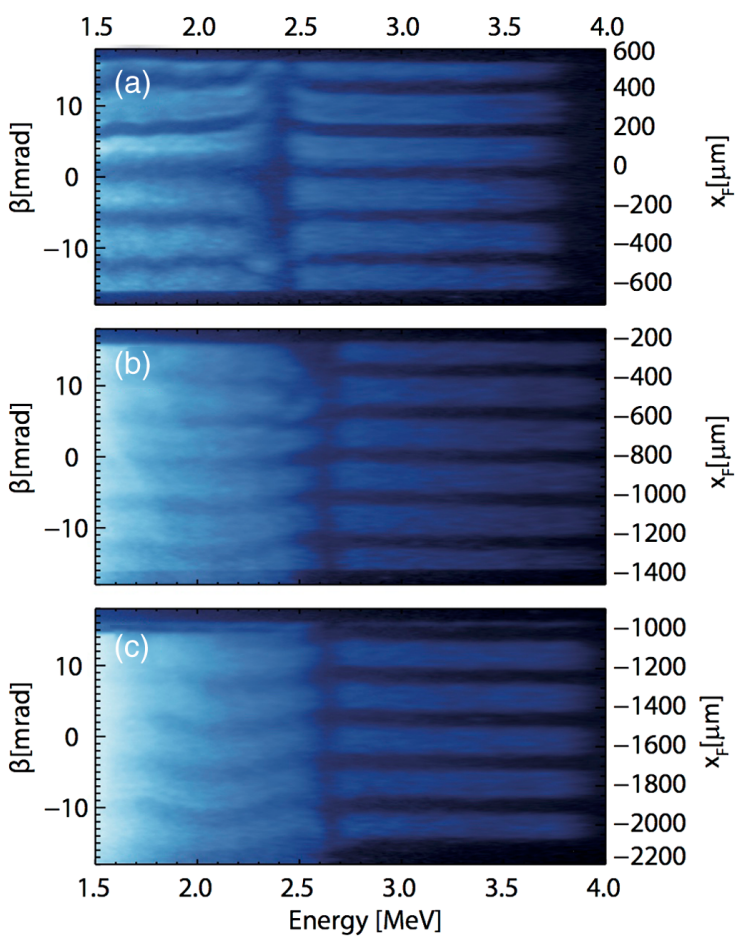

FIG. 5. Successive shots while probing in longitudinal geometry and varying the $\mathrm{x}_{F}$ parameter. The probe timing $\left(t_{\text {pump }}\right)$ corresponds to the proton t-pump energies $\epsilon_{\text {pump }}=2.4 \mathrm{MeV}$ (a) and $\epsilon_{\mathrm{pump}}=2.6 \mathrm{MeV}[(\mathrm{b})$ and (c)], respectively.

deflection measurements shows that the deflection maximum is shifted to higher probing times $t_{p}$ with increasing distance $\left|x_{F}\right|$. Assuming the field of an expanding ion front causes the deflection maximum, the slope of this shift can be regarded as the expansion velocity of the front. In the case of Fig. 3(a) a velocity of $2.39 \times 10^{7} \mathrm{~m} / \mathrm{s}$ is deduced, which corresponds to a proton energy of $3.01 \mathrm{MeV}$. In the case of Fig. 8(c) the velocity $2.34 \times 10^{7} \mathrm{~m} / \mathrm{s}$ is obtained, which results in a proton energy of $2.87 \mathrm{MeV}$. A comparison with the associated proton energy spectra shows that these values are in good agreement with the measured cutoff energies (cf. Fig. 4), which confirms the assumption.

Figure 5(a) shows a selected streak deflectometry measurement in longitudinal imaging configuration. The time $t_{\text {pump }}$ was set in agreement with the proton t-pump energy $\epsilon_{\text {pump }}=2.4 \mathrm{MeV}$.

Slight changes of particle number and high energy cutoff are present in the proton probe beam from shot to shot. These changes do not degrade the emittance of the beam [visible in the noninfluenced high energy part in Figs. 3(c) and 3(d)]. Slight enhancement of the cutoff energy accompanies with a faint decrease of particle density in the traces. Different current densities of the probe beam (as e.g. visible in Fig. 3) are always at a level which allows one to use the response of single particles in the field for model calculation. The initial emission angle $\beta$ and the lateral focus distance $x_{F}$ are indicated on the left and right ordinate, respectively.

In order to visualize the influence of transversal force components (along the foil surface) a grating with 200LPI was used to intersect the probing proton beam. The resulting proton streaks [Fig. 5(a)] on the detector exhibit prominent density dips in the energy range of $2.4 \mathrm{MeV}$, which corresponds to the selected proton t-pump energy $\epsilon_{\text {pump. }}$. These dips can be explained as a consequence of the decelerating and accelerating action of the normal electric field component $E_{\mathrm{N}}$. Thus the action of the ambipolar electric field leads to a redistribution of the energies within the probing proton beam [12]. In agreement with this interpretation, the dips are most pronounced near the center of the interaction $\left(\left|x_{F}\right|=0 \mu \mathrm{m}\right)$.

In addition, a bending of the proton streaks is visible at both sides of the dips, indicating the presence of radial electric field components $E_{\mathrm{R}}$ and/or the influence of a magnetic field B. On both sides the bending is directed outwards in respect to a line through the interaction center $\left(\left|x_{F}\right|=0 \mu \mathrm{m}\right)$, however the deflection is stronger at the low energy side.

At greater distances to the interaction center the outward deflection of the protons decreases. As a result no bending of the streaks is detectable for $\left|x_{F}\right| \gtrsim 600 \mu \mathrm{m}$ at the highenergy side of the dips. This observation is supported by additional measurements where the position of the pump focus was displaced in the $\mathrm{x}$-direction. This series of measurements is shown in Fig. 5. It shows that overlapping regions in the scan are well reproduced within the successive shots and that such parameter scans due to improved laser pulse characteristics are really possible.

\section{MODEL APPROACHES}

In the following three theoretical models are proposed, which provide different analytical descriptions of laserinduced electric and magnetic fields on thin foils. For convenience these models are referred to as model A, model B and model C. In principle model A and model B are variants of already published model descriptions [1315]. These descriptions have been extended and developed further in this work in order to explain the specific characteristic features of the streak deflectometry measurements in longitudinal and transversal imaging configuration. Model $\mathrm{C}$ is basically a combination of these two models and allows for an extensive and accurate reproduction of all experimental results. The models use calculated parameters (e.g. temperature, Debye length) from basic plasma equations with input of laser intensity and target density. Additionally free variables (e.g. decay constants) are used (four in model A, two in model B and thus six in model C, cf. the complete set in Table I) in a trial and error mode to calculate deflectograms which approximate the experiment. 


\section{A. Model A}

Model A is based on a published analytical model description [13]. It describes the electric and magnetic field distribution, which is generated on an ultrathin foil $(<1 \mu \mathrm{m})$ if an ultrashort $(<50 \mathrm{fs})$ laser pulse irradiates the target surface at an angle of $45^{\circ}$. The model accounts for the generation of hot electrons and their motion through the target (along the target normal, z-axis) as well as their transverse expansion along the target surface (x-axis) in a two-dimensional Cartesian geometry. Model A uses the precondition that a certain amount of laser energy is transferred into a population of fast (hot) electrons which appear at the cold rear side of the target foil initiating a positive charge and a respective strong field. The principle of charge neutralization drives strong restoring forces and a fast dynamic of the charge populations sets in. This and resulting fields are calculated. Therefore model A is applicable to effects which rely on strong and fast evolving fields being closely attached to the target surface.

The field dynamics depends on the evolution of the positively charged region, which is induced on the target surface and governed by the charge conservation law. The expansion of hot electrons is balanced by a flow of cold electrons from the target periphery in the direction of the charged region. As a result the normalized spot size $L_{n}(t)$ of the induced positive charge expands in the transverse direction depending on the target conductivity $\sigma_{\text {cold }}$ and on the effective electron collision frequency $\nu_{\mathrm{ef}}$. Its temporal evolution is described by the expression

$$
L_{n}(t)=\sqrt{L_{n 0}^{2}+\frac{\kappa}{\nu_{\mathrm{ef}}} \cdot\left[1-\exp \left(-\nu_{\mathrm{ef}} \omega_{\mathrm{pef}} t\right)\right]}
$$

where $L_{n 0}=D_{e 0} / r_{D 0}$ and $\kappa=\left(\sigma_{\text {cold }} \Delta a\right) /\left(\omega_{\text {pef }} r_{D 0}\right)$. The initial spot size in real space is denoted with $D_{e 0}$ and the thickness of the polymer foil with $\Delta a$. The (initial) plasma frequency of hot electrons is indicated by $\omega_{\text {pef }}$ and can be calculated depending on the initial density of hot electrons using the formula $\omega_{\text {pef }}=\sqrt{n_{e 0} e^{2} / \varepsilon_{0} m_{e}}$. Here $m_{e}$ is the mass and $e$ the charge of an electron. The initial Debye radius has the symbol $r_{D 0}$ and depends both on the density $n_{e 0}$ and the temperature $T_{e}$ of hot electrons using the equation $r_{D 0}=\sqrt{\varepsilon_{0} k_{B} T_{e} / e^{2} n_{e 0}}$.

The net positive charge density on the foil $\rho(x, z)$ is given by $\rho(x, z)=e n_{e 0} \eta_{e 0} \rho_{\eta}\left(x_{n}\right) \delta\left(z_{n}\right)$ where the coordinates $x_{n}=x / r_{D 0}$ and $z_{n}=z / r_{D 0}$ are normalized to the Debye radius of hot electrons $r_{D 0}$. The sharp boundary of the surface charge in target normal direction is modeled using the Dirac-delta function $\delta\left(z_{n}\right)$. The transverse profile of the charge density is described by a one-dimensional normalized distribution function $\rho_{\eta}\left(x_{n}\right)$ multiplied with the normalization factor $\eta_{e 0}=2 \pi D_{e 0} / r_{D 0}$. In this paper a normalized Lorentzian distribution is applied,

$$
\rho\left(x_{n}, t\right)=\frac{1}{\pi S_{L} L_{n}(t)} \cdot\left[1+\left(\frac{\left|x_{n}\right|}{S_{L} L_{n}(t)}\right)^{2}\right]^{-1},
$$

where the constant factor $S_{L}(\in[0,1])$ determines the (initial) width of the distribution in relation to $L_{n}(t)$.

Under the assumptions that the energy transfer between hot electrons and ions follows the adiabatic law and that the electron inertia can be neglected in the standard force equation for the density the twodimensional Poisson equation can be solved [16]. Using the modified Bessel function of second kind $K_{0}$ the electric potential $\phi\left(x_{n}, z_{n}, t\right)$ can be evaluated. The resulting formula

$$
\phi\left(x_{n}, z_{n}, t\right)=E_{0} \frac{\eta_{e 0}}{4 \pi} \int \rho\left[\tilde{x}_{n}, L_{n}(t)\right] K_{0}\left(\sqrt{\frac{z_{n}^{2}}{2}+\frac{x_{n}-\tilde{x}_{n}}{2}}\right) d \tilde{x}_{n},
$$

where $E_{0}=\sqrt{2 I_{L} / \varepsilon_{0} c}$, allows for the deduction of the electric and magnetic field distribution. However, these expressions also depend on modified Bessel functions, which make them unsuitable for particle simulations due to the long calculation time. For this reason the normal electric field component $E_{z}$, the transversal electric field component $E_{x}$ and the magnetic field component $B_{y}$ are described with the suitable approximations,

$$
\begin{gathered}
E_{x}(x, z, t)=-\sqrt{2} \frac{\pi U_{0} D_{e}}{r_{D}{ }^{2}} \frac{\partial \rho}{\partial x_{n}}\left(\frac{x}{r_{D}}, t\right) \cdot H\left(\left|\frac{z}{r_{D}}\right|-\frac{\Delta a}{2 r_{D}}\right) \cdot \exp \left[-\left(\left|\frac{z}{r_{D}}\right|-\frac{\Delta a}{2 r_{D}}\right) / \sqrt{2}\right], \\
E_{z}(x, z, t)=-\frac{\pi U_{0} D_{e}}{r_{D}^{2}} \rho\left(\frac{x}{r_{D}}, t\right) \cdot H\left(\left|\frac{z}{r_{D}}\right|-\frac{\Delta a}{2 r_{D}}\right) \cdot \operatorname{sgn}\left(\frac{z}{r_{D}}\right) \cdot \exp \left[-\left(\left|\frac{z}{r_{D}}\right|-\frac{\Delta a}{2 r_{D}}\right) / \sqrt{2}\right], \\
B_{y}(x, z, t)=-\frac{2 \pi^{2} \sqrt{2}}{c} \frac{U_{0} D_{e}}{r_{D}^{2}} \rho\left(\frac{x}{r_{D}}, t\right) \cdot v_{L}(t) \cdot H\left(\left|\frac{z}{r_{D}}\right|-\frac{\Delta a}{2 r_{D}}\right) \cdot \operatorname{sgn}\left(\frac{x}{r_{D}}\right) \cdot \operatorname{sgn}\left(\frac{z}{r_{D}}\right) \cdot \exp \left[-\left(\left|\frac{z}{r_{D}}\right|-\frac{\Delta a}{2 r_{D}}\right) / \sqrt{2}\right],
\end{gathered}
$$


where $U_{0}=k_{B} T_{e} / e, \operatorname{sgn}(x)$ is the signum-function and $H(z)$ the Heaviside step function [10]. Fields are calculated for distances greater than the Debye length. This results in sufficiently large arguments of the modified Bessel function which justifies an exponential approximation.

The proposed field functions [Eqs. (4), (5) and (6)] have been applied to explain the streak deflectometry measurements of Figs. 8(a)-8(c). Equations (4)-(6) have been multiplied with the additional factor $\exp \left(-t / \tau_{\text {ced }}\right)$ in order to account for the charge equilibration on a time scale $\tau_{\text {ced }} \approx 100$ ps. Thus global charge neutralization of the target foil is considered at a late stage of system development. Implementing implicitly the temporal function to the field expressions and not the charge development simplified the model calculation.

Furthermore $v_{L}(t)$ was defined by $v_{L}(t)=\left(1 / \omega_{\text {pef }}\right)$. $d L(t) / d t$ and $D_{e}=D_{e 0}=L_{n}(0) r_{D 0}$, as well as $r_{D}=r_{D 0}$ were applied in the conducted particle simulations. Using these expressions it became possible to reproduce the streak deflectometry measurement in longitudinal imaging configuration [Fig. 8(a)]. However, accurate results were only obtained by assuming relatively high values for the hot electron temperature $T_{e}$ in the order of $13 \mathrm{MeV}$ and by setting the initial radius of the electron spot size $D_{e}$ to a value $>10 \mu \mathrm{m}$. This is relatively high in comparison to the radius of the laser spot size $r_{L} \approx 1.5 \mu \mathrm{m}$ (FWHM).

This example is illustrated in Fig. 6 which shows that model fits of single deflectograms are also possible with unrealistic parameters. Different probing geometries circumvent this problem and serve as a kind of a benchmark. The quality of our proposed simple models and also more advanced computer simulations with their inherent parameter ambiguities is not sufficient to motivate numerical multiparametric fitting routines. Therefore we restrict our

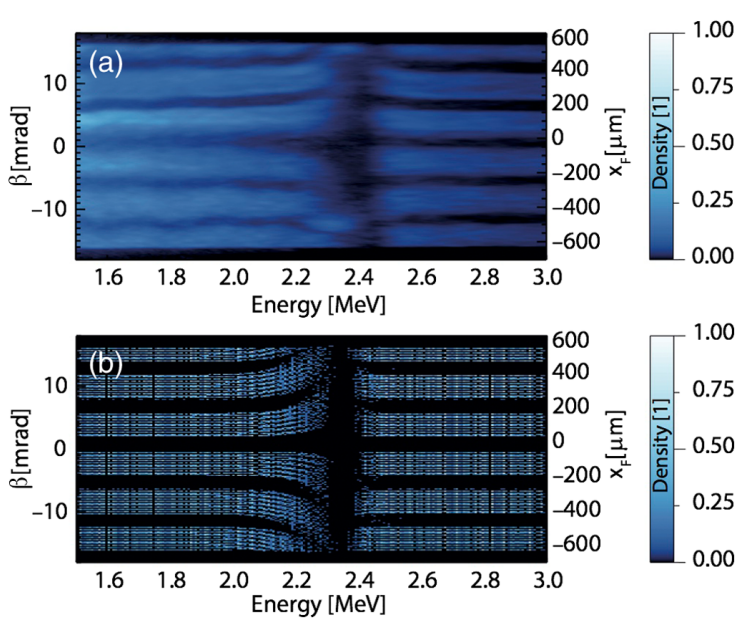

FIG. 6. Measured deflectogram (a) in longitudinal probing geometry and model simulation (b) with an unrealistic high electron temperature of $13 \mathrm{MeV}$ and other adapted parameters (model C variation without temporal development of the Debye length). parameter determination on a coincident visual impression (e.g. gap size, amplitude of deflection and functional form) between experimental and model picture.

In addition it was not possible to explain both the longitudinal and transversal measurements with one parameter set on the basis of Eqs. (4)-(6). In order to achieve a more comprehensive explanation for the experimental results in both imaging configurations the proposed model [13] has been modified and developed further in this paper. This extended version will be referred to as model $\mathrm{A}$ in the following. The principle idea of model $\mathrm{A}$ is to find an approximation for the temporal evolution of the Debye length $r_{D}(t)$ and to include this time dependence into Eqs. (4)-(6).

The approach developed here connects the expansion of the electron spot size

$$
D_{e}(t)=L_{n}(t) r_{D 0}
$$

with the temporal evolution of the hot electron density

$$
n_{e}(t)=\frac{N_{e}}{\pi D_{e}(t)^{2}} \frac{1}{2 r_{D}(t)},
$$

where $N_{e}$ is the number of hot electrons in a cylinder with the length $2 r_{D}(t)$ and the radius $D_{e}(t)$. Using the definition of the Debye radius $r_{\mathrm{D}}(t)=\sqrt{\varepsilon_{0} k_{B} T_{e} / e^{2} n_{e}(t)}$ Eq. (8) can be rearranged:

$$
n_{e}(t)=\frac{N_{e}^{2} e^{2}}{4 \pi^{2} \varepsilon_{0} k_{B} T_{e} D_{e}(t)^{4}}
$$

and the time dependence of the Debye length can be expressed as

$$
r_{D}(t)=r_{D 0}\left(\frac{D_{e t}(t)}{D_{e 0}}\right)^{2} .
$$

In the framework of model A Eqs. (4)-(6) multiplied with the decay factor $\exp \left(-t / \tau_{\text {ced }}\right)$ are used for the field description. However, the time dependence of the spot size as well as temporal evolution of the Debye radius are included in these equations via $D_{e}=D_{e}(t)$ [Eq. (7)] and $r_{D}=r_{D}(t)$ [Eq. (10)], respectively. In order to make model A applicable for multidimensional particle simulations its field description is extended to three spatial dimensions, by assuming cylindrical symmetry. Thus the necessary 3Dparticle tracking in the field is possible. The extension of the 2D-plane $(\mathrm{x}, \mathrm{z})$ model to a $3 \mathrm{D}(\mathrm{r}, \mathrm{z})$ geometry is possible if the terms connected to $(\mathrm{d} / \mathrm{rdr})$ can be omitted. Used functional dependencies are within this range. As a result the normal electric field component $E_{N(A)}(r, z, t)=$ $E_{z}(r, z, t)$, the radial electric field component $E_{R(A)}(r, z, t)=E_{x}(r, z, t)$ and the toroidal magnet field $B_{T(A)}(r, z, t)=B_{y}(r, z, t)$, with $r=\sqrt{x^{2}+y^{2}}$. The spatial 

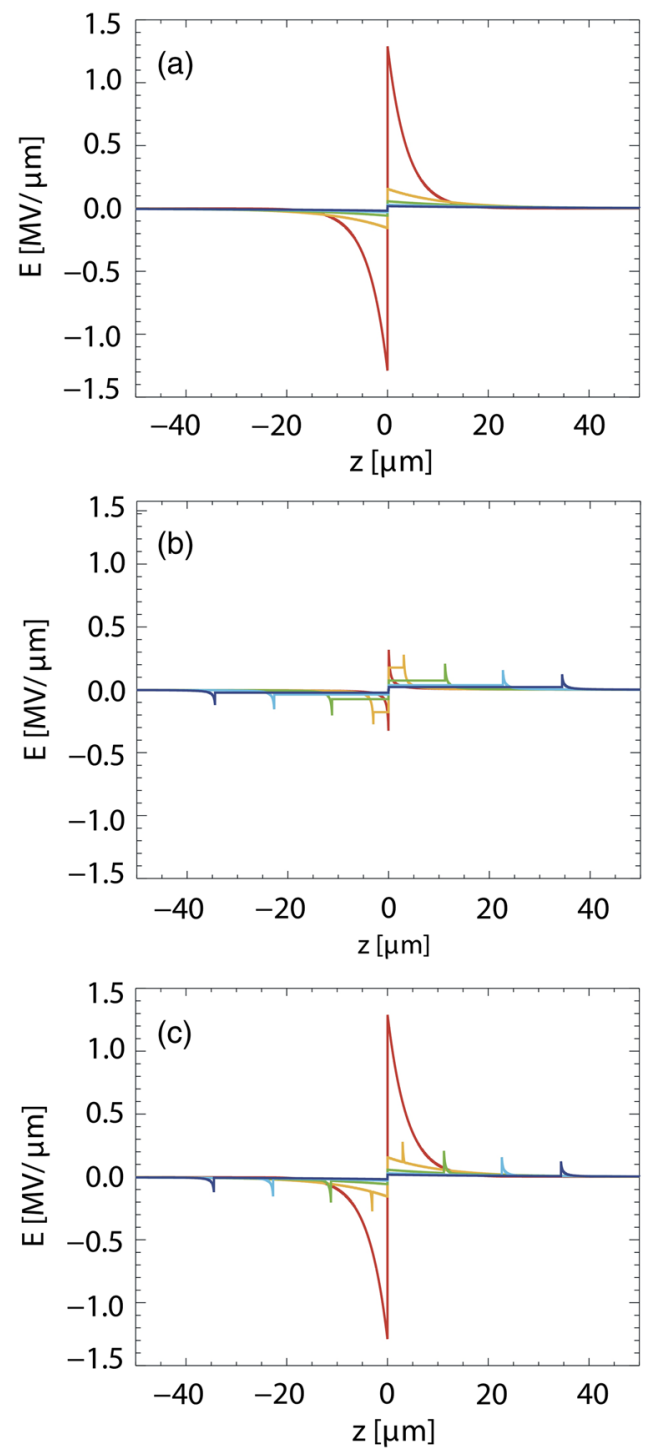

FIG. 7. Spatial distribution of the normal electric field component $E_{N}(r=0, z, t)$ in the z-direction at different times (red: $0 \mathrm{ps,}$ orange: 0.5 ps, green: 1 ps, light blue: 1.5 ps, dark blue: 2 ps. For the field calculations the parameters of Tables I and II have been used: (a) model A, (b) model B, (c) model C.

dependence of $E_{N(A)}(r, z, t)$ in the z-direction (at $r=0$ ) and its temporal evolution is illustrated in Fig. 7(a).

\section{B. Model B}

Model B provides an analytical description of the electric field structure of a moving ion front. It is based on a onedimensional model [14] that describes the isothermal plasma expansion into vacuum and explains the ion acceleration as the result of charge separation. In between a positively and a preceding negatively charged region an electric field is created, which peaks at a definite position. Since the accelerated ions accumulate at this position, an ion front is generated, which propagates with increasing velocity.
The spatial dependence of the field structure is described by a constant plateau region followed by a steep rise up to the front field peak and a decay of the expanding Gaussianshaped front. As an approximation for the spatial decay of the front region a function with the proportionality [17] $E_{\text {front }}(z) \propto\left(1+z / l_{s}\right)^{-1}$ is used, where the parameter $l_{s}$ determines the characteristic spatial scale of the decay. The electric field amplitudes of the front peak $E_{f}$ and the plateau region $E_{p}$, respectively, depend on time and are described by the formulas $[10,17]$

$$
\begin{gathered}
E_{f}=\frac{2 \mathcal{E}_{c}}{\sqrt{2 e_{m}+t^{2} / \tau_{i}^{2}}}, \\
E_{p}=\frac{2 \mathcal{E}_{c}}{\sqrt{2 e_{m}}+t^{2} / \tau_{i}^{2}}, \\
\mathcal{E}_{c}=\sqrt{\frac{n_{e 0} k_{B} T_{e}}{\varepsilon_{0}}},
\end{gathered}
$$

where $\tau_{i}=1 / \omega_{p i}$ represents the reciprocal value of the ion plasma frequency $\omega_{p i}=\sqrt{n_{e 0} Z e^{2} / m_{i} \varepsilon_{0}}$ and determines the characteristic scale of the temporal decay. The time dependent position of the ion front, which separates the plateau and front region, is described [14] by

$z_{\text {front }}=2 \sqrt{2 e_{m}} r_{D 0}\left\{\left[\tau \ln \left(\tau+\sqrt{\tau^{2}+1}\right)+\sqrt{\tau^{2}+1}\right]+1\right\}$,

where $\tau=t /\left(2 e_{m} \tau_{i}\right)$ and $e_{m}=\exp (1)$. In this isothermal description the plasma is described with one electron temperature $T_{e}$ and does not cool down while expanding. For this reason the model intrinsically overestimates the velocity of the ion front, which has been shown experimentally $[15,18]$. In addition the front velocity $v_{\text {front }}$ diverges for $t \rightarrow \infty$, which can be derived from Eq. (14). Therefore a maximum front velocity $v_{f, \max }$ is introduced [10], which limits the acceleration $v_{\text {front }} \leq$ $v_{f, \max }$ and can be determined experimentally.

Model B only provides a description for the electric field component $E_{N}$ normal to the target surface (z-direction), but considers neither radial electric field components nor magnetic fields. So far only a one-dimensional spatial dependence of the field structure $E_{N(B)}(z, t)$ is proposed here. In order to use model $\mathrm{B}$ in multidimensional particle simulations the field description is extended to three spatial dimensions [15], by assuming a Gaussian decay of the field amplitude $E_{N(B)}(r, z, t)=E_{N(B)}(z, t) \cdot \exp \left[-\left(r / l_{r}\right)^{2}\right]$ in the radial direction, where $r=\sqrt{x^{2}+y^{2}}$. The z-dependence of $E_{N(B)}(r, z, t)$ (at $\left.r=0\right)$ and its temporal evolution is shown in Fig. 7(b). 


\section{Model C}

Model $\mathrm{C}$ is proposed in this work. It is a combination of model $\mathrm{A}$ and model $\mathrm{B}$ and provides an analytical field description, which can reproduce the measured features of both imaging configurations.

In model $\mathrm{C}$ the radial electric field component $E_{R(C)}(r, z, t)$ and the toroidal magnetic field are $B_{T(C)}(r, z, t)$ are evaluated in the same way as in model A. However, for the calculation of the normal field component $E_{N(C)}(r, z, t)$ either model $\mathrm{A}$ or model $\mathrm{B}$ is used depending on two selection rules. First, within the plateau region $\left(z<z_{\text {front }}\right)$ the description always follows model A. In other words the equation $E_{N(C)}(r, z, t)=E_{N(A)}(r, z, t)$ applies if $z<z_{\text {front }}$ [cf. Eq. (14)]. Second, in the front region $\left(z \geq z_{\text {front }}\right)$ the description of model A is only used if the field amplitude of model $\mathrm{A}$ is higher than the amplitude of model $\mathrm{B}$, which means if $E_{N(A)}(r, z, t)>E_{N(B)}(r, z, t)$. This dependence is shown in Fig. 7 illustrating the z-dependence of $E_{N(C)}(r, z, t)($ at $r=0)$ at different moments in time. As a result the different field components of model $\mathrm{C}$ can be summarized using the equations

$$
\begin{aligned}
E_{N(C)}(r, z, t)= & \begin{cases}E_{N(A)}(r, z, t), & z<z_{\text {front }} \\
\max \left[E_{N(A)}(r, z, t), E_{N(B)}(r, z, t)\right], & z \geq z_{\text {front }}\end{cases} \\
& E_{R(C)}(r, z, t)=E_{R(A)}(r, z, t), \\
& B_{T(C)}(r, z, t)=B_{T(A)}(r, z, t) .
\end{aligned}
$$

\section{SIMULATION AND DISCUSSION}

In order to test the applicability range of the presented models and to draw conclusions on the laser-induced fields three-dimensional particle simulations have been
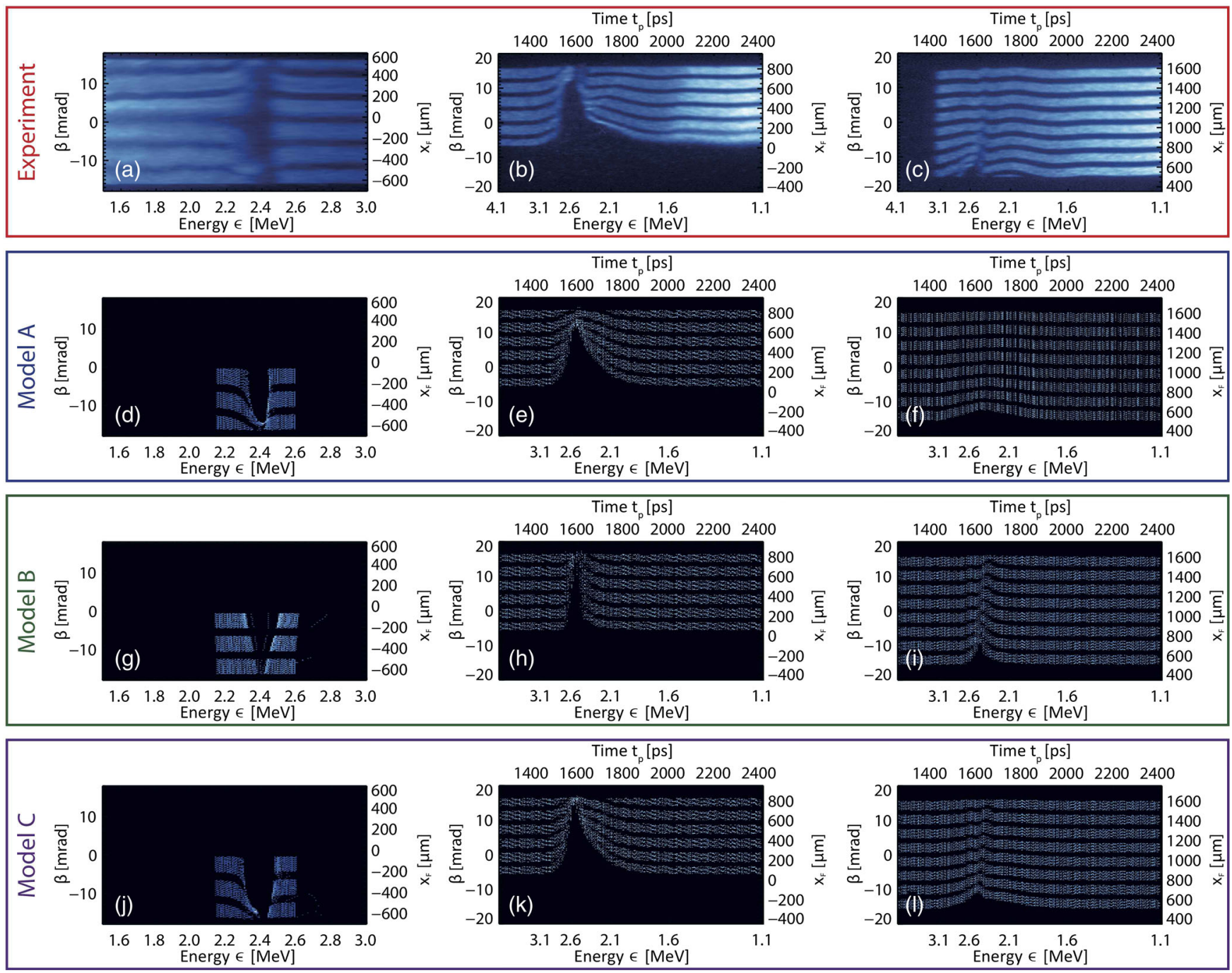

FIG. 8. Experimental [(a)-(c)] and simulated [(d)-(l)] streak deflections in longitudinal [(a), (d), (g), (j)] and transversal [(b), (c), (e), (f), (h), (i), (k), (l)] imaging configuration. 
conducted. For this purpose a particle tracer [19] has been used to calculate the trajectories of protons that propagate through a simulated streak deflectometry [10] including the field distribution according to one of the proposed model descriptions.

In the case of model $\mathrm{A}$ the determination of the parameters $D_{e 0}, T_{e}, N_{e}, \sigma_{\text {cold }}, \nu_{\text {ef }}$ and $\tau_{\text {ced }}$ enables the calculation of the fields on the basis of Eqs. (1)-(10). The radius of the initial electron spot size $D_{e 0}$ was set to the value of the laser waist size $r_{L}$ (the radius where the intensity decreases by a factor of $e^{-2}$ against the maximum value). The mean laser intensity within this radius is denoted by $I_{L}$ and was used for the calculation of the ponderomotive potential $\phi_{p}$, which allows for an estimation of the hot electron temperature $k_{B} T_{e} \approx \phi_{p}\left(I_{L}\right)$ [20]. The initial number of hot electrons $N_{e}$ in the cylinder with the length $2 r_{D}(t)$ and the radius $D_{e}(t)$ [cf. Eq. (8)] can be estimated via the formula $N_{e}=\eta_{\mathrm{L}} \epsilon_{\mathrm{L}} /\left(k_{B} T_{e}\right)$, where $\epsilon_{\mathrm{L}}=I_{L} \pi r_{L}{ }^{2} \tau_{L}$ is the laser energy and $\eta_{\mathrm{L}}$ the absorption coefficient. $\eta_{\mathrm{L}}$ refers to the effective absorption into hot electrons which are responsible for the created acceleration potential. This is only a small part of the overall electron population and therefore this value is low (cf. Table I.) Also it is not directly accessible via measurements. It is indirectly justified from measured secondary effects like the observed emission of fast ions. The choice of the value is in accordance to other theoretical modeling and simulation [13].
The conductivity of cold electrons $\sigma_{\text {cold }}$ is set to a value of approximately $\omega_{\text {pec }} / 4 \pi$ [13], where $\omega_{\text {pec }}$ is the cold electron frequency and depends on the cold electron density $n_{\mathrm{ec}}$ via $\omega_{\mathrm{pec}}=\sqrt{n_{e c} e^{2} / \varepsilon_{0} m_{e}}$. In addition to the choice of the one-dimensional normalized distribution function $\rho_{\eta}\left(x_{n}, S_{L}, t\right)$ (Lorentz distribution with an initial normalized length $S_{L}$ ) the parameters $\eta_{L}, \nu_{\text {ef }}$ and $\tau_{\text {ced }}$ (cf. Table I) are treated as free variables in order to achieve a good reproduction of the experiment, resulting in an effective number of four free parameters. The applied criterion is a subjective visible coincidence between model picture and measurement. Based on the applied model variables and Eqs. (1)-(10) relevant field and plasma parameters were calculated. All variables and parameters are summarized in Table I. The simulation results using model A are depicted in Figs. 8(d)-8(f). The comparison to the experiment reveals that model A allows for an accurate reproduction of the streak deflections in both imaging configurations.

Using model A with the parameters of Table I, a magnetic field amplitude of over $10^{4} \mathrm{~T}$ is obtained. However, the influence of the magnetic field on the proton deflection is negligible compared to the impact of the radial electric field.

Model A can also explain the measured cutoff energies of protons (between 2.4 and $3.0 \mathrm{MeV}$ ) [10] that originate directly from the polymer foil. If the acceleration of a single proton in the field distribution of model A is calculated

TABLE I. Variables and simulation parameters of model A and model C.

\begin{tabular}{|c|c|c|c|}
\hline Parameter & Description & Value & Unit \\
\hline$D_{e 0}$ & Spot size of initial charge & 4 & $\mu \mathrm{m}$ \\
\hline$T_{e}$ & Hot electron temperature & 1.05 & $\mathrm{MeV}$ \\
\hline$N_{e}$ & Number of hot electrons & $2.15 \times 10^{9}$ & 1 \\
\hline$\sigma_{\text {cold }}$ & Target conductivity for cold electrons & $7.36 \times 10^{14}$ & $1 / \mathrm{s}$ \\
\hline$\nu_{\mathrm{ef}}$ & Effective electron collision frequency & $1 \times 10^{-4}$ & 1 \\
\hline$\tau_{\text {ced }}$ & Temporal charge neutralization & 75 & Ps \\
\hline$\phi_{p}$ & Electron ponderomotive potential & $1.68 \times 10^{-13}$ & $\mathrm{~J}$ \\
\hline$\phi_{p, \mathrm{MeV}}$ & Electron ponderomotive energy & 1.05 & $\mathrm{MeV}$ \\
\hline$r_{D 0}$ & Initial Debye radius & 2.72 & $\mu \mathrm{m}$ \\
\hline$\eta_{L}$ & Effective absorption into hot electrons & $8 \times 10^{-4}$ & 1 \\
\hline$I_{L}$ & Laser intensity in focus & $1.8 \times 10^{19}$ & $\mathrm{~W} / \mathrm{cm}^{2}$ \\
\hline$\epsilon_{L}$ & Laser pulse energy & 0.452 & $\mathrm{~J}$ \\
\hline$\tau_{L}$ & Laser pulse duration & 50 & fs \\
\hline$r_{L}$ & Laser spot (focus) radius & 4 & $\mu \mathrm{m}$ \\
\hline$\omega_{\text {pec }}$ & Cold electron plasma frequency & $1.26 \times 10^{16}$ & $1 / \mathrm{s}$ \\
\hline$n_{\mathrm{ec}}$ & Cold electron density & $5 \times 10^{22}$ & $1 / \mathrm{cm}^{3}$ \\
\hline$L_{n 0}$ & Initial spot size (normalized) of charge & 1.47 & 1 \\
\hline$\kappa$ & Extension factor for spot size of charge & $5.13 \times 10^{-2}$ & 1 \\
\hline$\omega_{\text {pef }}$ & Hot electron plasma frequency & $1.58 \times 10^{14}$ & $1 / \mathrm{s}$ \\
\hline$n_{e 0}$ & Initial hot electron density & $7.86 \times 10^{18}$ & $1 / \mathrm{cm}^{3}$ \\
\hline$\Delta a$ & Thickness of target foil & 30 & $\mathrm{Nm}$ \\
\hline$S_{L}$ & Initial width of charge distribution & 0.3 & 1 \\
\hline$U_{0}$ & Initial field potential & 1.05 & MV \\
\hline
\end{tabular}


TABLE II. Variables and simulation parameters of model B and model C.

\begin{tabular}{lccc}
\hline \hline Parameter & Description & Value & Unit \\
\hline$T_{e}$ & Hot electron temperature & 1.05 & $\mathrm{MeV}$ \\
$\tau_{i}$ & Decay time for ion front acceleration & 350 & $\mathrm{fs}$ \\
$\omega_{p i}$ & Ion plasma frequency & $3.69 \times 10^{12}$ & $1 / \mathrm{s}$ \\
$v_{f, \max }$ & Maximum ion front velocity & $2.35 \times 10^{9} \mathrm{~cm} / \mathrm{s}$ \\
$l_{r}$ & Radial decay of electric field & 400 & $\mu \mathrm{m}$ \\
$l_{s}$ & Decay of field in ion front & 0.25 & $\mu \mathrm{m}$ \\
\hline \hline
\end{tabular}

numerically, using the parameters of Table I, a final energy of $2.6 \mathrm{MeV}$ is obtained. However model A fails to reproduce the shift of the deflection maximum to higher probing times $t_{p}$ with increasing distance $\left|x_{F}\right|$, which has been observed experimentally [cf. Figs. 8(c) and 8(f)] for larger distances $\left(\left|x_{F}\right| \gtrsim 700 \mu \mathrm{m}\right)$.

Regarding model B, the parameters $T_{e}, \tau_{i}, v_{f, \max }, l_{r}$ and $l_{s}$ must be determined to calculate the normal electric field component $E_{N(B)}(r, z, t)$ within the particle simulation. The hot electron temperature $T_{e}$ is approximated via $k_{B} T_{e} \approx$ $\phi_{p}\left(I_{L}\right)$ and the characteristic decay time $\tau_{i}$ is set to a value of approximately $1 / \omega_{p i}$. The maximal expansion velocity $v_{f, \max }$ is determined on the basis of the perturbation of the deflection maximum in Fig. 8(c), which propagates in space and time and has a slope that represents the expansion velocity. In order to achieve a good agreement of the simulation with the proton streak deflectometry measurements of Figs. 8(a)-8(c) the two free parameters $l_{r}$ and $l_{s}$ (cf. Table II) have been varied.

All variables and model parameters are listed in Table II. As the simulation results show, model B is not suited to explain the measured streak deflections in longitudinal imaging configuration [cf. Fig. 8(g)]. Also in transversal configuration [Figs. 8(h) and 8(i)] the agreement between experiment and simulation is not very accurate. Nonetheless model B can qualitatively explain the temporal evolution of the observed streak perturbations in Fig. 8(i) at larger distances $\left(\left|x_{F}\right| \gtrsim 700 \mu \mathrm{m}\right)$, which affirms the assumption that these perturbations are caused by the field of a moving proton front.

Figures $8(j)-8(1)$ show the calculated streak deflections when the field description of model $\mathrm{C}$ is applied within the simulation. Apparently the proposed combination of model A and model B [Eqs. (15)-(17)] enables an extensive and accurate reproduction of the experimental results [Figs. 8(a)-8(c)]. Using model C, not only single and selected measurements can be reproduced by the particle simulation, but also (and at the same time) measurements with varied experimental parameters and measurements that were obtained using different imaging configurations. Since model C is a combination of model A and model B it has in principal six free parameters (four in model A, two in model B). However, it should be stressed that for the here shown simulation results of model $\mathrm{C}$ these parameters have not been varied (again). Instead the same values were used that were determined before by varying the free parameters of model A and model B to approximate the experimental results independently in both cases. The simple superposition of models A and B works well because they account for different field dynamics in respect to complementary spatial regions. This is in principle a consequence of the temporal development of the overall process. Model A reflects well the strong field initialization at the surface but omits specific field propagation due to the initialized ion movement. Model B explains the resulting field dynamics at later times and in extended regions correctly but clearly underestimates the start potential at the target surface. Thus the combination of both models is straightforward. It became directly obvious when complementary geometrical setups had been applied in field imaging.

\section{PICOSECOND EXPERIMENTS}

Figures 9(a) and 9(b) show two processed streak deflectometry measurements in longitudinal imaging configuration. Both measurements were performed using identical experimental parameters (cf. experimental setup). However in the case of Fig. 9(a) the position of the grating compressor, which provided the shortest pulse length, was changed by a distance of $10 \mathrm{~mm}$. As a result, a pulse length $\tau_{L}$ of approximately $2.7 \mathrm{ps}$ was generated, leading to an averaged intensity level within the beam waist of $3.3 \times$ $10^{17} \mathrm{~W} / \mathrm{cm}^{2}\left[5.8 \times 10^{17} \mathrm{~W} / \mathrm{cm}^{2}\right.$ (FWHM) $]$. In addition the variation of the grating position introduced a time delay between the pump and probe laser pulse, which resulted in a change of the associated t-pump energy $\epsilon_{\text {pump }}$ from 2.4 to 1.227 MeV.

Both in the femtosecond case [Fig. 9(b)] and in the picosecond case [Fig. 9(a)] prominent density gaps appear in the recorded proton streaks within the energy range around the value of the t-pump energy $\epsilon_{\text {pump. }}$ This can be explained by the decelerating and accelerating effect of an ambipolar electric field around the polymer foil, which leads to a redistribution of the proton energies around $\epsilon=\epsilon_{\text {pump }}$ [12]. Thus, a clear allocation between $\epsilon$ and $t_{p}$ is

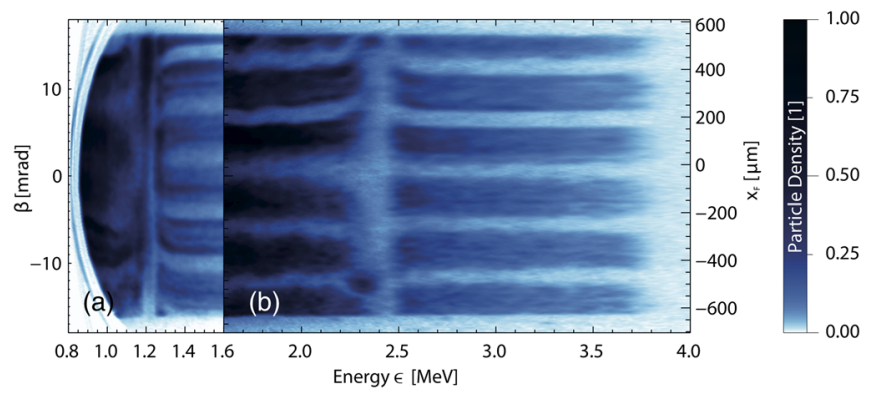

FIG. 9. Comparison between long-pulse-induced (a) and short-pulse-induced (b) streak deflections. 
not possible. Nevertheless, in principle higher proton energies correspond to lower probing times and thus reflect an earlier stage of the field evolution at the polymer foil.

In the picosecond case, the proton streaks at the low energy side of the gaps appear broadened, deformed and blurred, making the interpretation difficult. However, at the high-energy side a clear bending of the proton streaks becomes visible in the form of a focusing effect. Here the streak deflections are directed inwards, that is towards the center of the laser plasma interaction at $\left|x_{F}\right|=0$.

To see this, one should keep in mind that the mean initial emission angle of a single trace can be deduced by its position at energies $\epsilon \gg \epsilon_{\text {pump }}$ (cf. Sec. III). In the case of Fig. 9(a) the energy $\epsilon=1.6 \mathrm{MeV}$ allows for a relatively accurate estimation. At lower energies $(\epsilon \approx 1.25 \mathrm{MeV})$ the absolute values of the mean propagation angles (of the streaks) are clearly reduced in comparison to their initial emission angles $\beta$.

In contrast, the proton streak deflections in the femtosecond case are exactly opposite, pointing outwards.

The induced and controllable change of the deflection direction of protons within a certain energy interval is a significant experimental finding. Regarding the picosecond case, the application of a Thomson slit spectrometer allowed for a clear determination of the magnetic field configuration at the rear side of the polymer foil.

Depending on its kinetic energy, each probing proton has a position either before $(z<a)$ or behind $(z>a)$ the polymer foil [cf. Figs. 2 and 10], when the deflecting fields are initiated at the time $t_{\text {pump }}$. Due to the definition of the t-pump energy $\epsilon_{\text {pump }}$ (cf. Sec. III) all protons with energies $\epsilon>\epsilon_{\text {pump }}$ have already passed the polymer foil and are located at a position behind the rear side of the foil $(z>a)$ at the time $t_{p}=t_{\text {pump. }}$.

In the picosecond case, the inward bending of the proton streaks at the high energy side of the gap just appears within the energy range $\epsilon>\epsilon_{\text {pump. }}$. Therefore, these protons reflect the exclusive influence of the extended fields and the associated force acting on the rear side of the foil. Conversely, this means that none of these protons are influenced by the electric and magnetic field distribution acting at the front side of the polymer target.

From PIC simulation we know that fields located at the rear and the front side of the target do not extend to the

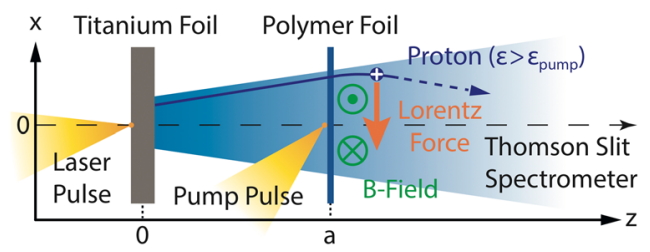

FIG. 10. Deflection of a single proton at the rear side of the polymer foil due to the influence of the $(\vec{v} \times \vec{B})$-term of the Lorentz force. opposite side. This situation has been used in all our model approaches and it is illustrated in Fig. 10 showing the direction of the acting Lorentz force on a proton behind the rear side of the foil $(z>a)$ at the time $t_{p}=t_{\text {pump }}$. Due to the charge up of a thin foil during a laser plasma interaction the radial electric field component must point outward. This is just opposite to the marked direction of the net force that leads to the observed focusing effect. Therefore, the radial electric field component allows no explanation for the measured inward deflection. Quite different field scenarios might appear especially in the case of longer laser pulses and/or significantly higher laser energy impact together with a certain prepulse level as other results [21] suggest. Our parameter situation is different and also the creation of long (ns) lasting, much stronger and more extended fields would result in quite different deflectograms as those shown in Fig. 9.

In principle also the longitudinal electric field could be responsible for a change of the propagation direction. After the acceleration process at the titanium foil each proton has a certain initial ejection angle $\beta=\tan \left(v_{x} / v_{z}\right)$ depending on its longitudinal and transversal velocity components. If the proton is reaccelerated by the longitudinal electric field of the polymer foil its longitudinal velocity component can be increased while its radial velocity component remains constant. This leads to a reduction of the ratio $\left|v_{x} / v_{z}\right|$ and thus of the absolute value of the measured angle $\beta$. In principle this would lead to an inward bending of the proton traces.

However, based on the measurement in Fig. 9(a) one can estimate that this mechanism is not sufficient to explain the observed proton defection quantitatively. If one regards, for instance, the lower edge of second proton trace from above, one can deduce that $\beta$ changes from its initial value of $\sim 9$ mrad to a value of $\sim 7 \mathrm{mrad}$. In addition an effective energy gain $\Delta \epsilon$ of approximately $0.05 \mathrm{MeV}$ can be deduced from the width of the energy gap.

Given a proton with the initial energy $\epsilon_{0}=\epsilon_{\text {pump }}=$ $1.227 \mathrm{MeV}$ and the initial ejection angle $\beta_{0}=9 \mathrm{mrad}$, one can calculate the initial longitudinal velocity $v_{z 0}=$ $1.532 \times 10^{7} \mathrm{~m} / \mathrm{s}$ and transversal velocity component $v_{x 0}=1.379 \times 10^{5} \mathrm{~m} / \mathrm{s}$. After the interaction the proton has the energy $\epsilon_{1}=\epsilon_{0}+\Delta \epsilon \approx 1.277 \mathrm{MeV}$ and the longitudinal velocity component $v_{z 1}$ has a value of $\sim 1.563 \times 10^{7} \mathrm{~m} / \mathrm{s}$. Assuming that the transversal velocity component $v_{x 1}$ remains unchanged during the interaction $\left(v_{x 1}=v_{x 0}\right)$, one can estimate that the propagation angle $\beta_{1}$ after the interaction has an approximate value of $8.824 \mathrm{mrad}$. The change of the emission angle can be calculated by $\Delta \beta=\beta_{0}-\beta_{1}$ and has the value $\sim 0.176 \mathrm{mrad}$, which cannot explain the observed change of $\sim 2 \mathrm{mrad}$.

During the imaging series with long pump pulses we did not compensate the delay which was introduced by the increased grating distance to stretch the pulse. This resulted in a $t_{\text {pump }}$ for probing protons with lower (around 1.2 MeV 
instead of $2.5 \mathrm{MeV}$ ) kinetic energy and it set an observation window with a safety margin concerning available cutoff energies.

Spectrometer images of the intersected probe beam penetrating the cold target without pumping show clear undisturbed stripes throughout the energy window between 0.9 and $2.5 \mathrm{MeV}$. Independently, in former experiments we tested probing of energy redistribution with different probing energies of $1.2,1.5$ and $2.5 \mathrm{MeV}$. Concluding we assume that the probe beam behaves similar with the kinetic energy as the essential parameter. Stopping and scattering is more pronounced for slower protons which is visible from enhanced blurring of the picture [Fig. 9(a)] formed by protons $<1.2 \mathrm{MeV}$ and interacting with the pumped plasma state. The characteristic and emphasized inward bending of traces stems from protons with $>1.2 \mathrm{MeV}$ kinetic energy. They have passed the cold target and are influenced by a field initiated at $t_{\text {pump }}$.

A change of the trajectory by $2 \mathrm{mrad}$ visible around $1.2 \mathrm{MeV}$ proton energy should translate to a change of $(1.2 / 2.5)^{1 / 2} * 2 \mathrm{mrad} \sim 1.4 \mathrm{mrad}$ around $2.5 \mathrm{MeV}$. A significant feature cannot appear with completely different bending [cf. Fig. 9(b)] in the case of probing with 2.5 or 1.2 $\mathrm{MeV}$ protons. This also supports that the observed effect is really due to different pumping and resulting field configuration and not due to possible changes in the probe beam. The lateral target extension of $5 \mathrm{~mm}$ in diameter is due the target foil coverage of a mask plate with $5 \mathrm{~mm}$ holes. Initiated electron currents and following charging produce electric fringe fields from edges which can in principle cause focusing beam effects. Such a situation should also become visible with short pump pulses which we do not detect even when displacing the probe beam $2 \mathrm{~mm}$ out of the center and probing closer to the edge at higher fringe field strength. This encourages the interpretation of an acting magnetic field although a last ultimate proof cannot be given.

The $(\vec{v} \times \vec{B})$-term of the Lorentz force must be responsible for the observed phenomenon and the direction of the acting force in Fig. 10. Hence, the term must be dominant against the radial electric field component, which means that $|\vec{v} \times \vec{B}|>\left|\vec{E}_{R}\right|$, assuming a similar effective extension of the two fields at about $\pm 1 \mathrm{~mm}$ from the original center. The start extension of the calculated magnetic field is depicted in Fig. 11. It accounts for the observed deflection.

In addition, the polarity of the laser induced toroidal magnetic field must be consistent with the marked direction in Fig. 10, otherwise a defocusing effect would occur. This phenomenon is observed for the first time in connection with high intensity laser pulses $\left(I_{L}>10^{17} \mathrm{~W} / \mathrm{cm}^{2}\right)$ with high temporal contrast and solid ultrathin foil targets. In recent investigations [22,23] of the magnetic field structure generated on thin foils using high intensity lasers $\left(I_{L} \sim 10^{19} \mathrm{~W} / \mathrm{cm}^{2}\right)$ just the opposite magnetic polarity
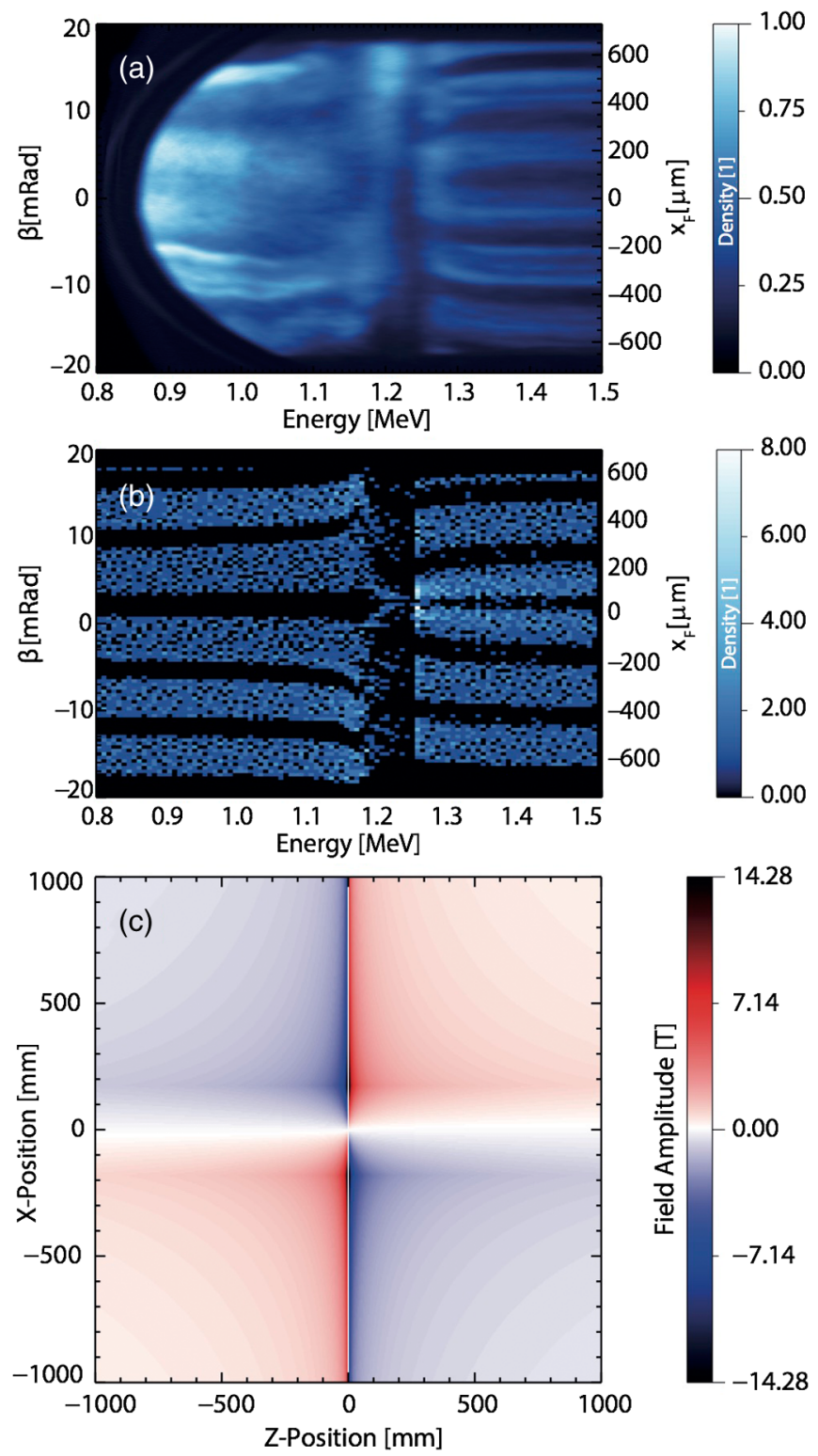

FIG. 11. Proton deflectogram: (a) experiment, (b) model calculation obtained for a ps-pulse irradiation of a $30 \mathrm{~nm}$ plastic foil in a longitudinal probing geometry, (c) calculated magnetic field accounting for the deflection.

was found. However, this polarity (i.e. opposite to the marked direction in Fig. 10) is also in agreement with the analytical description of model A, explaining the longitudinal streak deflection measurements in connection with femtosecond laser pulses.

In the case of laser irradiated thin foils, the polarity of the thermoelectric magnetic field generation [24-27] is just the opposite to the observed polarity in the picosecond case as illustrated in Fig. 10. This indicates that another mechanism is responsible for the magnetic field generation. In principle the observed magnetic field polarity is possible due to dc currents in steep density gradients driven by temporal variations of the ponderomotive force [28-30]. Another 
explanation is the occurrence of hot electron currents, which are either directed into the target [31,32] or along the target surface [33-35]. So far magnetic fields with a polarity opposite to the orientation of a thermoelectric field were only observed experimentally in the interaction of relativistic intense laser pulses that propagate in a preionized plasma [36]. This raises the question if an undercritical plasma could be generated due to the picosecond pulse interaction.

The observed proton deflection pattern of Fig. 11(a) at the high-energy side of the gap can be quantitatively reproduced with a numerical particle simulation [10]. The simulation result confirms the measured polarity of the magnetic field at the rear side of the target. The B-field is calculated at the time of its initialization [Fig. 11(b)]. The used electron temperature is $25 \mathrm{keV}$. For simplicity we changed only this parameter. Using the same effective absorption this results in a higher electron number and a respective current which builds the magnetic field being able to account for the measured deflection. At this stage of investigation conclusions about possible changes of other parameters are not possible. Compared to the femtosecond case the temperature is approximately at a similar factor lower as it is the driving laser intensity due to the similarly prolonged laser pulse. The chosen decay time of the field is 50 ps. The simulated deflectogram (based on model A) [cf. Fig. 11(b)] compares well to the measurement.

However, a clear determination of the magnetic field generation mechanism is not possible. Therefore, further theoretical research is required to find the physical mechanism that leads to the creation of the observed magnetic field.

\section{CONCLUSION}

In this paper we studied the dynamics and structure of the electric and magnetic fields that were generated on ultrathin polymer foils $(30-50 \mathrm{~nm})$ when irradiated with high intensity femtosecond $\left(10^{19}-10^{20} \mathrm{~W} / \mathrm{cm}^{2}\right)$ and picosecond $\left(\sim 10^{17} \mathrm{~W} / \mathrm{cm}^{2}\right)$ laser pulses using ultrahigh laser contrast $\left(10^{10}-10^{11}\right)$. For this purpose the proton streak deflectometry method has been applied and developed further. We could show that the use of ultrahigh contrast laser pulses significantly increased the reproducibility of experiments and improved the accuracy of the applied imaging method in comparison to former experiments. Further advancement was realized by applying the method in two different imaging configurations. It enabled us to gather complementary information about the investigated field structure. In particular the influence of different field components (parallel and normal to the target surface) and the impact of a moving ion front could be studied in detail. Regarding the experiments with femtosecond laser pulses these particular measured features could be explained using two different models providing an analytical description of the laser induced fields. Their ability to reproduce the streak deflectometry measurements was tested on the basis of three-dimensional particle simulations. Simulating the experiment in two different imaging configurations expanded our knowledge of the application range and limitations of the single models and enabled the further development of the investigated analytical model descriptions itself. A modification and combination of the two models allowed for an extensive and accurate reproduction of the experimental results in both imaging configurations. This way, not only single and selected measurements could be reproduced by the particle simulation, but also (and at the same time) measurements with varied experimental parameters and measurements that were obtained using different imaging configurations. In this regard, the described methodological approach might offer a new path for a comprehensive reconstruction of the spatial and temporal field distribution of laser-plasma interactions.

In addition, we could demonstrate that a controlled change of the pulse duration can be used to manipulate the propagation direction of the proton beam within a certain energy range. The change from 50 femtosecond to 2.7 picosecond laser pulses (with the same energy content) led to a transition of the dominating force acting on the probing proton beam at the rear side of the polymer foil. In the picosecond case the $(\vec{v} \times \vec{B})$-term of the Lorentz force dominated over the action of the $\vec{E}$-field and was responsible for the direction of the acting force. The applied imaging method allowed for an unambiguous determination of the magnetic field polarity at the rear side of the ultrathin foil, revealing an unexpected orientation of the field that is just opposite to the polarity of the thermoelectric magnetic field generation.

\section{ACKNOWLEDGMENTS}

The research leading to these results has received funding from Deutsche Forschungsgemeinschaft within the program CRC/Transregio 18 and from LASERLABEUROPE (Grant Agreement No. 284464, EC's Seventh Framework Program). A. A. A. acknowledges the provided computation resources of JSC at Project No. HBU15. P. V. N. acknowledges the support by the Institute for Basic Science under IBS-R012-D1.

[1] H. Daido, M. Nishiuchi, and A. S. Pirozhkov, Review of laser-driven ion sources and their applications, Rep. Prog. Phys. 75, 056401 (2012).

[2] S. P. Hatchett et al., Electron, photon, and ion beams from the relativistic interaction of Petawatt laser pulses with solid targets, Phys. Plasmas 7, 2076 (2000).

[3] J. Braenzel, C. Pratsch, P. Hilz, C. Kreuzer, M. Schnürer, H. Stiel, and W. Sandner, Note: Thickness determination of 
free-standing ultra-thin foils using a table top laboratory extreme ultraviolet source, Rev. Sci. Instrum. 84, 056109 (2013).

[4] M. A. Spivack, Parylen thin films for radiation applications, Rev. Sci. Instrum. 41, 1614 (1970).

[5] T. Sokollik et al., Transient electric fields in laser plasmas observed by proton streak deflectometry, Appl. Phys. Lett. 92, 091503 (2008).

[6] T. Ceccotti, A. Lévy, H. Popescu, F. Réau, P. D’Oliveira, P. Monot, J. P. Geindre, E. Lefebvre, and Ph. Martin, Proton Acceleration with High-Intensity Ultrahigh-Contrast Laser Pulses, Phys. Rev. Lett. 99, 185002 (2007).

[7] M. Schnürer et al., Comparison of femtosecond laser-driven proton acceleration using nanometer and micrometer thick target foils, Laser Part. Beams 29, 437 (2011).

[8] M. Borghesi et al., Electric field detection in laser-plasma interaction experiments via the proton imaging technique, Phys. Plasmas 9, 2214 (2002).

[9] M. P. Kalashnikov, H. Schönnagel, and W. Sandner, High temporal contrast front end with $\mathrm{CaF} 2$-based XPW temporal filter for high intensity lasers, AIP Conf. Proc. 1465, 13 (2012).

[10] F. Abicht, Doctoral dissertation, Technische Universität, Berlin, 2015, https://depositonce.tu-berlin.de/handle/ $11303 / 4881$.

[11] S. Ter-Avetisyan, M. Schnürer, and P. V. Nickles, Time resolved corpuscular diagnostics of plasmas produced with high-intensity femtosecond laser pulses, J. Phys. D 38, 863 (2005).

[12] F. Abicht, J. Braenzel, G. Priebe, Ch. Koschitzki, A. A. Andreev, P. V. Nickles, W. Sandner, and M. Schnürer, Tracing ultrafast dynamics of strong fields at plasmavacuum interfaces with longitudinal proton probing, Appl. Phys. Lett. 105, 034101 (2014).

[13] A. Andreev, T. Ceccotti, A. Levy, K. Platonov, and P. Martin, Divergence of fast ions generated by interaction of intense ultra-high contrast laser pulses with thin foils, New J. Phys. 12, 045007 (2010).

[14] P. Mora, Plasma Expansion into a Vacuum, Phys. Rev. Lett. 90, 185002 (2003).

[15] M. Amin, Doctoral dissertation, Heinrich-Heine-Universität, Düsseldorf, 2008, http://docserv.uni-duesseldorf.de/servlets/ DocumentServlet?id=10395.

[16] A. Andreev, Generation and Application of Super-Strong Laser Fields (Nova Science Publishers, Hauppauge, NY, 2001).

[17] L. Romagnani et al., Dynamics of Electric Fields Driving the Laser Acceleration of Multi-MeV Protons, Phys. Rev. Lett. 95, 195001 (2005).

[18] J. Fuchs et al., Laser-driven proton scaling laws and new paths towards energy increase, Nat. Phys. 2, 48 (2006).

[19] O. J. Luiten, S. B. van der Geer, M. J. de Loos, F. B. Kiewiet, and M. J. van der Wiel, How to Realize Uniform Three-Dimensional Ellipsoidal Electron Bunches, Phys. Rev. Lett. 93, 094802 (2004).
[20] S. C. WIilks, W. L. Kruer, M. Tabak, and A. B. Langdon, Absorption of Ultra-Intense Laser Pulses, Phys. Rev. Lett. 69, 1383 (1992).

[21] Y. Paudel et al., Self-proton/ion radiography of laserproduced proton/ion beam from thin foil targets, Phys. Plasmas 19, 123101 (2012).

[22] G. Sarri et al., Dynamics of Self-Generated, Large Amplitude Magnetic Fields Following High-Intensity Laser Matter Interaction, Phys. Rev. Lett. 109, 205002 (2012).

[23] W. Schumaker et al., Ultrafast Electron Radiography of Magnetic Fields in High-Intensity Laser-Solid Interactions, Phys. Rev. Lett. 110, 015003 (2013).

[24] J. A. Stamper, K. Papadopoulos, R. N. Sudan, S. O. Dean, E. A. McLean, and J. M. Dawson, Spontaneous Magnetic Fields in Laser-Produced Plasmas, Phys. Rev. Lett. 26, 1012 (1971).

[25] A. R. Bell et al., Observation of plasma confinement in picosecond laser-plasma interactions, Phys. Rev. E 48, 2087 (1993).

[26] F. N. Beg, A. R. Bell, A. E. Dangor, C. N. Danson, A. P. Fews, M. E. Glinsky, B. A. Hammel, P. Lee, P. A. Norreys, and M. Tatarakis, A study of picosecond laser-solid interactions up to $10^{19} \mathrm{~W} \mathrm{~cm}^{-2}$, Phys. Plasmas 4, 447 (1997).

[27] C. A. Cecchetti et al., Magnetic field measurements in laser-produced plasmas via proton deflectometry, Phys. Plasmas 16, 043102 (2009).

[28] R. N. Sudan, Mechanism for the Generation of $10^{9} \mathrm{G}$ Magnetic Fields in the Interaction of Ultraintense Short Laser Pulse with an Overdense Plasma Target, Phys. Rev. Lett. 70, 3075 (1993).

[29] V. K. Tripathi and C. S. Liu, Self-generated magnetic field in an amplitude modulated laser filament in a plasma, Phys. Plasmas 1, 990 (1994).

[30] R. J. Mason and M. Tabak, Magnetic Field Generation in High-Intensity-Laser-Matter Interactions, Phys. Rev. Lett. 80, 524 (1998).

[31] A. Pukhov and J. MeyerTerVehn, Laser Hole Boring into Overdense Plasma and Relativistic Electron Currents for Fast Ignition of ICF Targets, Phys. Rev. Lett. 79, 2686 (1997).

[32] A. R. Bell and R. J. Kingham, Resistive Collimation of Electron Beams in Laser-Produced Plasmas, Phys. Rev. Lett. 91, 035003 (2003).

[33] F. Brunel, Anomalous absorption of high intensity subpicosecond laser pulses, Phys. Fluids 31, 2714 (1988).

[34] P. Gibbon, Efficient Production of Fast Electrons from Femtosecond Laser Interaction with Solid Targets, Phys. Rev. Lett. 73, 664 (1994).

[35] H. Ruhl and P. Mulser, Relativistic Vlasov simulation of intense fs laser pulse-matter interaction, Phys. Lett. A 205, 388 (1995).

[36] M. Borghesi, A. J. Mackinnon, R. Gaillard, O. Willi, A. Pukhov, and J. Meyer-ter-Vehn, Large Quasistatic Magnetic Fields Generated by a Relativistically Intense Laser Pulse Propagating in a Preionized Plasma, Phys. Rev. Lett. 80, 5137 (1998). 